JOURNAL OF THE

AMERICAN MATHEMATICAL SOCIETY

Volume 18, Number 3, Pages 685-710

S 0894-0347(05)00487-X

Article electronically published on April 25, 2005

\title{
THE ESSENTIALLY TAME LOCAL LANGLANDS CORRESPONDENCE, I
}

\author{
COLIN J. BUSHNELL AND GUY HENNIART
}

To the memory of Albrecht Fröhlich

1. Let $F$ be a non-Archimedean local field; we assume throughout that the residue field $\mathbb{k}_{F}$ of $F$ is finite, of characteristic $p$. Let $\mathcal{W}_{F}$ be the Weil group of $F$, formed relative to some separable algebraic closure of $F$. For each integer $n \geqslant 1$, let $\mathcal{G}_{n}^{0}(F)$ be the set of equivalence classes of irreducible smooth (complex) representations of $\mathcal{W}_{F}$ of dimension $n$. Likewise, let $\mathcal{A}_{n}^{0}(F)$ be the set of equivalence classes of irreducible supercuspidal representations of the group $\mathrm{GL}_{n}(F)$. The Langlands correspondence gives a bijection

$$
{ }_{F} \mathcal{L}_{n}: \mathcal{G}_{n}^{0}(F) \stackrel{\approx}{\rightarrow} \mathcal{A}_{n}^{0}(F)
$$

for each $n$. The existence of the family $\left\{{ }_{F} \mathcal{L}_{n}\right\}$ is established indirectly, by methods relying heavily on geometric or global constructions [21, [14, [15]; explicit information about it, of the sort essential for local calculations, is inconveniently hard to obtain. Indeed, in virtually no case is there a complete, explicit account.

2. In a series of papers, of which this is the first, we describe ${ }_{F} \mathcal{L}_{n}$ in the "essentially tame" case. For $\sigma \in \mathcal{G}_{n}^{0}(F)$, let $t(\sigma)$ be the number of unramified characters $\chi$ of $\mathcal{W}_{F}$ for which $\chi \otimes \sigma \cong \sigma$. The integer $t(\sigma)$ divides $n$, and we say that $\sigma$ is essentially tame if $p$ does not divide $n / t(\sigma)$. (This is equivalent to demanding that the restriction of $\sigma$ to the wild inertia subgroup of $\mathcal{W}_{F}$ be a sum of characters: see Appendix (A.4) below.) We denote by $\mathcal{G}_{n}^{\text {et }}(F)$ the set of essentially tame classes $\sigma \in \mathcal{G}_{n}^{0}(F)$. Analogously, we say that $\pi \in \mathcal{A}_{n}^{0}(F)$ is essentially tame if $p$ does not divide $n / t(\pi)$, where $t(\pi)$ is the number of unramified characters $\chi$ of $F^{\times}$such that $\pi$ is equivalent to the representation

$$
\chi \pi: g \longmapsto \chi(\operatorname{det} g) \pi(g) .
$$

Let $\mathcal{A}_{n}^{\text {et }}(F)$ denote the set of essentially tame $\pi \in \mathcal{A}_{n}^{0}(F)$. The correspondence (1) then restricts to a bijection

$$
{ }_{F} \mathcal{L}_{n}^{\mathrm{et}}: \mathcal{G}_{n}^{\mathrm{et}}(F) \stackrel{\approx}{\rightarrow} \mathcal{A}_{n}^{\mathrm{et}}(F)
$$

Our aim is to give a complete and explicit description of the map (2).

Received by the editors March 29, 2004.

2000 Mathematics Subject Classification. Primary 22E50.

Key words and phrases. Explicit local Langlands correspondence, base change, automorphic induction, tame lifting, admissible pair.

Much of the work in this programme was carried out while the first-named author was visiting, and partly supported by, l'Université de Paris-Sud. Both authors were also partially supported by the EU network "Arithmetical Algebraic Geometry".

(C)2005 American Mathematical Society Reverts to public domain 28 years from publication 
When $p \nmid n$, we have $\mathcal{G}_{n}^{\text {et }}(F)=\mathcal{G}_{n}^{0}(F)$ and $\mathcal{A}_{n}^{\text {et }}(F)=\mathcal{A}_{n}^{0}(F)$. Under this hypothesis (and when $F$ has characteristic zero), Moy [23] (see also Reimann [24]) wrote down a bijection $\mathcal{G}_{n}^{0}(F) \rightarrow \mathcal{A}_{n}^{0}(F)$ which, at the time, seemed a good candidate for the Langlands correspondence. We show in this paper that Moy's correspondence is, in its general form, quite close to the Langlands correspondence; in later papers, for example [7, we will see that the two diverge significantly at the level of detail. (However, Moy's map is the Langlands correspondence when $n$ is prime.)

3. One classifies the elements of $\boldsymbol{\mathcal { G }}_{n}^{\text {et }}(F)$ using admissible pairs. We recall the matter briefly: see the Appendix for more details.

Definition. Let $E / F$ be a finite, tamely ramified field extension and let $\xi$ be a quasicharacter of $E^{\times}$. The pair $(E / F, \xi)$ is called admissible if it satisfies the following two conditions. Let $K$ range over intermediate fields, $F \subset K \subset E$.

(1) If $\xi$ factors through the relative norm $\mathrm{N}_{E / K}$, then $K=E$.

(2) If $\xi \mid U_{E}^{1}$ factors through $\mathrm{N}_{E / K}$, then $E / K$ is unramified.

Let $P_{n}(F)$ denote the set of $F$-isomorphism classes of admissible pairs $(E / F, \xi)$ in which $[E: F]=n$. The map

$$
\begin{aligned}
P_{n}(F) & \longrightarrow \mathcal{G}_{n}^{\mathrm{et}}(F), \\
(E / F, \xi) & \longmapsto \operatorname{Ind}_{E / F} \xi,
\end{aligned}
$$

then provides a canonical bijection. (Here, we regard $\xi$ as a quasicharacter of $\mathcal{W}_{E}$ via class field theory, and denote by $\operatorname{Ind}_{E / F}$ the functor of induction from representations of $\mathcal{W}_{E}$ to representations of $\mathcal{W}_{F}$ : see (A.3) below for more details.)

We combine the structure theory for supercuspidal representations [9] with the technique of tame lifting of simple characters [3] to construct a canonical bijection

$$
\begin{aligned}
P_{n}(F) & \longrightarrow \mathcal{A}_{n}^{\text {et }}(F), \\
(E / F, \xi) & \longmapsto{ }_{F} \pi_{\xi} .
\end{aligned}
$$

Together, (3) and (4) yield a canonical bijection

$$
{ }_{F} \mathcal{N}_{n}: \mathcal{G}_{n}^{\mathrm{et}}(F) \longrightarrow \mathcal{A}_{n}^{\mathrm{et}}(F),
$$

which we call the "naïve correspondence". Our aim is to compare the maps ${ }_{F} \mathcal{N}_{F}$, ${ }_{F} \mathcal{L}_{n}^{\text {et }}$. The main result of this paper is:

Theorem A. Let $(E / F, \xi) \in P_{n}(F)$, and set $\sigma=\operatorname{Ind}_{E / F} \xi$. There is a tamely ramified character $\mu={ }_{F} \mu_{\xi}$ of $E^{\times}$such that

$$
{ }_{F} \mathcal{L}_{n}^{\mathrm{et}}(\sigma)={ }_{F} \pi_{\mu \xi}
$$

Evaluation of the character ${ }_{F} \mu_{\xi}$ in the general case requires the development of quite elaborate methods: in this paper, we treat it only in the following special case. Let $\delta_{E / F}$ denote the discriminant character of the extension $E / F$, that is, the determinant of the regular representation $\operatorname{Ind}_{E / F} 1$. We show:

Theorem B. Let $(E / F, \xi) \in P_{n}(F)$; suppose that $n$ is odd and $E / F$ is totally ramified. We then have

$$
{ }_{F} \mu_{\xi}=\delta_{E / F} \circ \mathrm{N}_{E / F} .
$$


4. Our general method is based on the following fundamental fact. Let $K / F$ be a finite, cyclic extension. We thus have the operations of restriction and induction connecting the representations of the Weil, or Weil-Deligne, groups of $K$ and $F$. Under the Langlands correspondence, these correspond respectively to base change $\mathrm{b}_{K / F}$ and automorphic induction $\mathrm{A}_{K / F}$. When $K / F$ is also tamely ramified, the theory of tame lifting [3, 6] describes how the classification from [9] interacts with these operations.

When we restrict to essentially tame representations, the compatibility with base change and automorphic induction is sufficient to determine the Langlands correspondence uniquely: we outline a proof at the end of $\S 3$.

In particular, the epsilon factor of pairs [19], [27] plays no rôle whatsoever. Indeed, it is not presently known whether the preservation of $L$ - and epsilon factors does determine the correspondence when restricted to essentially tame representations.

In the later papers of the series, we shall completely determine the character ${ }_{F} \mu_{\xi}$ of Theorem A. As we shall first see in [7, it takes a rather complicated form. Some of this is traceable to our construction of the map (4). At a certain critical point, it relies on a choice which, while both natural and canonical in the technical sense, is not the only one available. We have retained it for consistency with the literature, and for its simplicity and directness. On the other hand, some of the complexity in ${ }_{F} \mu_{\xi}$ seems unavoidable. We will return to this matter in a later paper, with more facts in hand.

5. We place our results in their historical context. We recall that, when $p \nmid n$, we have $\mathcal{G}_{n}^{\text {et }}(F)=\mathcal{G}_{n}^{0}(F)$ and $\mathcal{A}_{n}^{\text {et }}(F)=\mathcal{A}_{n}^{0}(F)$. In that case, Howe [18] first constructed the map (4) $P_{n}(F) \rightarrow \mathcal{A}_{n}^{\text {et }}(F)$, but he was only able to prove it is injective. Moy 23] then took up the problem and proved, under the hypothesis that $F$ has characteristic zero, that (4) is bijective. The restriction on characteristic was essential since his method relied on a description of the admissible dual of $D^{\times}$, for a central $F$-division algebra $D$ of dimension $n^{2}$ [11], [20], and on the existence of the Jacquet-Langlands correspondence. At that time, the latter was only available in characteristic zero [25]. We, on the other hand, can proceed much more directly and generally: the construction of the map (4) is an instance of the theory of tame lifting [3], 6] and its bijectivity follows from the classification theorems of [9].

One can modify the naïve correspondence by "tame twisting", to get bijections $P_{n}(F) \rightarrow \mathcal{A}_{n}^{\text {et }}(F)$ of the form $(E / F, \xi) \mapsto{ }_{F} \pi_{\nu_{\xi} \xi}$, where $\nu_{\xi}$ is a tame character of $E^{\times}$varying with $\xi$. Moy observed that one could choose the system $\xi \mapsto \nu_{\xi}$ so that the corresponding bijection $\mathcal{G}_{n}^{\text {et }}(F) \rightarrow \mathcal{A}_{n}^{\text {et }}(F)$ satisfies those properties of the Langlands correspondence known at the time he was writing: these mainly concern behaviour relative to $L$ - and epsilon factors of a single representation. In totality, these properties are rather weak. They do not imply that Moy's map is the Langlands correspondence, or even that the correspondence can be obtained at all as a tame twist of the naïve correspondence.

Theorem A finally confirms the accuracy of Moy's basic intuition, even if the fine details are different. It should be noted that Theorem A is, in effect, an instance of the main theorem (7.1) of [6], a result of considerable depth.

We mention that Bushnell and Fröhlich [2] took a similar approach to the representations of $D^{\times}$and came to similar conclusions: the Langlands correspondence seemed to be obtainable from the analogue of the naïve correspondence by a process 
of tame twisting, but the precise suggestions in [2] are inaccurate to very much the same degree as Moy's.

We point out the later paper of Reimann [24]. He showed that, after the correction of minor errors and tangential misconceptions, the approaches of [23], [2], could be accommodated within the same formal framework. The concision and accuracy of [24] make it a valuable consolidation of the earlier papers, but it gets no closer to the true correspondence than they.

Note on characteristic. The operations of base change and automorphic induction are constructed, in [1] and [16] respectively, on the hypothesis that $F$ has characteristic zero. The operation of tame lifting (of endo-classes of simple characters) [3] is defined in arbitrary characteristic. The main results of [3], which are basic to everything we do here, relate tame lifting to base change and automorphic induction. They depend on the standard character relations and a short list of other properties concerned with linear independence of supercuspidal characters on certain sets. As we remarked in [3] $\$ 16]$, a theory of base change and automorphic induction in positive characteristic, with these auxiliary properties, would instantly extend the main results of 3 to that case. Such a theory is constructed in [17], together with some useful refinements of the kind worked out in the appendices to [8]. The results of [6] depend only on those of [3], [8] along with certain conductor estimates valid in all characteristics. We therefore anticipate the outcome of 17 and proceed as if the results of [3, 6] hold in positive characteristic. This allows us here to treat all characteristics on an equal footing.

Notation and background. Throughout, $F$ is a non-Archimedean local field; we let $\mathfrak{o}_{F}$ be the discrete valuation ring in $F$ and $\mathfrak{p}_{F}$ the maximal ideal of $\mathfrak{o}_{F}$. We set $\mathbb{k}_{F}=\mathfrak{o}_{F} / \mathfrak{p}_{F}$ and $p=\operatorname{char} \mathbb{k}_{F}$. We write $U_{F}=\mathfrak{o}_{F}^{\times}$and $U_{F}^{n}=1+\mathfrak{p}_{F}^{n}, n \geq 1$. We let $v_{F}: F^{\times} \rightarrow \mathbb{Z}$ be the canonical additive valuation.

If $K / F$ is a finite field extension, we use similar notation relative to $K$ and we write $\mathrm{N}_{K / F}, \operatorname{Tr}_{K / F}$ for the relative norm and trace respectively.

We fix a character $\psi_{F}$ of $F$, trivial on $\mathfrak{p}_{F}$ but not on $\mathfrak{o}_{F}$.

If $\pi \in \mathcal{A}_{n}^{0}(F)$, we denote the contragredient of $\pi$ by $\check{\pi}$ and the central quasicharacter of $\pi$ by $\omega_{\pi}$.

a. Let $V$ be a finite-dimensional $F$-vector space and write $A=\operatorname{End}_{F}(V), G=$ $\operatorname{Aut}_{F}(V)$. We use the notation of [9] for simple strata in $A$ and simple characters in $G$. Thus, if $[\mathfrak{A}, l, 0, \beta]$ is a simple stratum in $A$, we have the compact open subgroups $H^{1}(\beta, \mathfrak{A})$ and $J^{i}(\beta, \mathfrak{A})$ of $G, i=0,1$, along with the set $\mathcal{C}\left(\mathfrak{A}, \beta, \psi_{F}\right)$ of simple characters of $H^{1}(\beta, \mathfrak{A})$. (We drop $\psi_{F}$ from the notation for much of the time, as in $[9]$.)

b. We shall require the notion of endo-equivalence for simple characters, and also the operation of tame lifting for endo-equivalence classes (or endo-classes, for short): this theory is developed in [3], but the summary in [6, §1] may be found helpful. We let $\mathcal{E}(F)$ denote the set of endo-classes of simple characters over $F$. If $[\mathfrak{A}, l, 0, \beta]$ is a simple stratum in $A$ and $\theta \in \mathfrak{C}(\mathfrak{A}, \beta)$, we write $\mathcal{E}_{F}(\theta)$ for the endo-class of $\theta$. We adjoin to $\mathcal{E}(F)$ a trivial element $\Theta_{0}$, which may be regarded as the class of trivial characters of groups $U_{\mathfrak{A}}^{1}$, where $\mathfrak{A}$ ranges over hereditary $\mathfrak{o}_{F}$-orders in matrix algebras over $F$. 
c. If $K / F$ is a finite, tamely ramified field extension, there is a canonical surjective map $\mathrm{R}_{K / F}: \mathcal{E}(K) \rightarrow \mathcal{E}(F)$, transitive in the extension $K / F$. If $\Theta \in \mathcal{E}(F)$, the

fibre $\mathrm{R}_{K / F}^{-1}(\Theta)$ is finite, and its elements are the $K / F$-lifts of $\Theta$. In particular, if $\Theta$ is the endo-class of $\theta \in \mathfrak{C}(\mathfrak{A}, \beta)$, the $K / F$-lifts of $\Theta$ are parametrized by the simple components of the algebra $F[\beta] \otimes_{F} K$.

d. Let $\pi \in \mathcal{A}_{n}^{0}(F)$. We attach to $\pi$ an endo-class $\Theta(\pi) \in \mathcal{E}(F)$ as follows. If $\pi$ has a fixed vector for the group $U_{\mathfrak{M}}^{1}, \mathfrak{M}=\mathrm{M}_{n}\left(\mathfrak{o}_{F}\right)$, we set $\Theta(\pi)=\Theta_{0}$ (one says that " $\pi$ has level zero"). Otherwise, $\pi$ contains a simple character $\theta \in \mathcal{C}(\mathfrak{A}, \beta)$, for some simple stratum $[\mathfrak{A}, n, 0, \beta]$. In this case, we set $\Theta(\pi)=\mathcal{E}_{F}(\theta)$.

We shall frequently need the following special cases of the main result (7.1) of 6]. Let $K / F$ be a finite, tamely ramified, cyclic extension, and let $\pi \in \mathcal{A}_{n}^{0}(F)$ and $\rho \in \mathcal{A}_{m}^{0}(K)$. First, if the base change $\pi_{K}=\mathrm{b}_{K / F}(\pi)$ is supercuspidal, then

$$
\Theta(\pi)=\mathrm{R}_{K / F}\left(\Theta\left(\pi_{K}\right)\right) .
$$

More precisely, $\Theta\left(\pi_{K}\right)$ is the unique $K / F$-lift of $\Theta(\pi)$. Second, if the automorphically induced representation $\rho^{K}=\mathrm{A}_{K / F}(\rho)$ is supercuspidal, then

$$
\Theta\left(\rho^{K}\right)=\mathrm{R}_{K / F}(\Theta(\rho)) .
$$

For the general statement, we refer to [6], although we shall only use the cases above.

\section{TAMELY RAMIFIED SIMPLE CHARACTERS}

Let $\Theta \in \mathcal{E}(F)$; there is a finite-dimensional $F$-vector space $V$, a simple stratum $[\mathfrak{A}, l, 0, \beta]$ in $\operatorname{End}_{F}(V)$, and a simple character $\theta \in \mathfrak{C}(\mathfrak{A}, \beta)$ such that $\Theta=\mathcal{E}_{F}(\theta)$. The quantities

$$
e(\Theta)=e(F[\beta] \mid F), \quad f(\Theta)=f(F[\beta] \mid F), \quad \operatorname{deg} \Theta=[F[\beta]: F],
$$

depend only on the endo-class $\Theta$, not the choice of representatives $\theta$ or $\beta$, 3, 8.11]. For the trivial element $\Theta_{0}$, we define $e\left(\Theta_{0}\right)=f\left(\Theta_{0}\right)=\operatorname{deg} \Theta_{0}=1$.

Let $\Theta \in \mathcal{E}(F)$; we say that $\Theta$ is tame or tamely ramified if $p$ does not divide $e(\Theta)$. We write $\mathcal{E}^{\text {et }}(F)$ for the set of tame endo-classes $\Theta \in \mathcal{E}(F)$ : in particular, $\mathcal{E}^{\text {et }}(F)$ contains the trivial element of $\mathcal{E}(F)$.

The aim of this section is to give an independent description of the set $\mathcal{E}^{\mathrm{et}}(F)$.

1.1. Let $K / F$ be a finite, tamely ramified field extension and let $\Theta \in \mathcal{E}^{\text {et }}(F)$. We say that $K / F$ splits $\Theta$ if $\Theta$ has a $K / F$-lift $\Theta_{K}$ such that $\operatorname{deg} \Theta_{K}=1$.

Proposition 1.1. (1) Let $\Theta \in \mathcal{E}^{\mathrm{et}}(F)$ and let $K / F$ split $\Theta$; then $\operatorname{deg} \Theta$ divides $[K: F]$.

(2) Let $\Theta \in \mathcal{E}^{\mathrm{et}}(F)$ have degree $d$. There then exists a tamely ramified extension $K / F$, of degree $d$, which splits $\Theta$. Moreover, $\Theta$ determines $K / F$ uniquely, up to F-isomorphism.

Proof. We choose a finite-dimensional $F$-vector space $V$ together with a simple stratum $[\mathfrak{A}, n, 0, \beta]$ in $\operatorname{End}_{F}(V)$ and a simple character $\theta \in \mathcal{C}(\mathfrak{A}, \beta)$ such that $\mathcal{E}_{F}(\theta)=\Theta$. By definition, $\operatorname{deg} \Theta=[F[\beta]: F]$ and $e(\Theta)=e(F[\beta] \mid F)$. In particular, the field extension $F[\beta] / F$ is tamely ramified.

Let $K / F$ be finite and tamely ramified; we write $K \otimes_{F} F[\beta]=\prod_{i} E_{i}$, where the $E_{i}$ are fields. Each $E_{i}$ is a tamely ramified extension of $K$, and the corresponding 
$K / F$-lift $\Theta_{i}$ of $\Theta$ satisfies $\operatorname{deg} \Theta_{i}=\left[E_{i}: K\right]$. Thus $K / F$ splits $\Theta$ if and only if $E_{i}=K$, for some $i$. This occurs if and only if there exists an $F$-embedding of $F[\beta]$ in $K$, so we have proved (1) and the existence statement of (2). Further, if $K / F$ has degree $d$ and splits $\Theta$, this embedding $F[\beta] \rightarrow K$ must be an isomorphism.

Let $K / F$ be tamely ramified and let $\Theta \in \mathcal{E}^{\text {et }}(F)$; we say that $K / F$ is a splitting field for $\Theta$ if it splits $\Theta$ and $[K: F]=\operatorname{deg} \Theta$. By the proposition, any $\Theta \in \mathcal{E}^{\text {et }}(F)$ has a splitting field, and this is unique up to $F$-isomorphism. Moreover, the proof of the proposition shows:

Corollary 1.1. Let $\Theta \in \mathcal{E}^{\mathrm{et}}(F)$ and let $K / F$ be a finite, tamely ramified field extension. Then $K / F$ splits $\Theta$ if and only if $K$ contains a splitting field for $\Theta$.

Remark. For the moment, let $\Theta \in \mathcal{E}(F)$ be arbitrary, that is, not necessarily tamely ramified. Choose a simple stratum $[\mathfrak{A}, l, 0, \beta]$ in $\operatorname{some~}_{\operatorname{End}}(V)$ such that $\Theta=\mathcal{E}_{F}(\theta)$ for some $\theta \in \mathcal{C}(\mathfrak{A}, \beta)$. The same argument as above shows that the maximal tamely ramified sub-extension of $F[\beta] / F$ is uniquely determined, up to $F$-isomorphism, by the endo-class $\Theta$. This is not the case for the wildly ramified part.

1.2. We make a simple observation. Let $\chi$ be a non-trivial character of $U_{F}^{1}$ : suppose $\chi$ is trivial on $U_{F}^{n+1}$ but not on $U_{F}^{n}$, for some $n \geq 1$. There then exists $c \in F$, with $v_{F}(c)=-n$, such that

$$
\chi(1+x)=\psi_{F}(c x), \quad 2 v_{F}(x) \geqslant n+1 .
$$

The quadruple $\left[\mathfrak{o}_{F}, n, 0, c\right]$ is a simple stratum in $\operatorname{End}_{F}(F)=F$ and $\chi$ is an element of $\mathcal{C}\left(\mathfrak{o}_{F}, c, \psi_{F}\right)$. We can therefore form the endo-class $\mathcal{E}_{F}(\chi) \in \mathcal{E}(F)$. If $\chi$ is the trivial character of $U_{F}^{1}$, then $\mathcal{E}_{F}(\chi)$ is the trivial endo-class. In this way, we can identify the dual $\widehat{U}_{F}^{1}$ of $U_{F}^{1}$ :

$$
\widehat{U}_{F}^{1}=\{\Theta \in \mathcal{E}(F): \operatorname{deg} \Theta=1\} .
$$

If $K / F$ is a finite tame extension and $\chi \in \widehat{U}_{F}^{1}$, the endo-class $\varepsilon_{F}(\chi)$ has a unique $K / F$-lift, namely $\mathcal{E}_{K}\left(\chi \circ \mathrm{N}_{K / F}\right)$. Put another way, if we write $\xi=\chi \circ \mathrm{N}_{K / F} \in \widehat{U}_{K}^{1}$, then

$$
\mathrm{R}_{K / F} \mathcal{E}_{K}(\xi)=\mathcal{E}_{F}(\chi) .
$$

1.3. We consider pairs $(K / F, \chi)$, where $K / F$ is a finite, tamely ramified field extension and $\chi \in \widehat{U}_{K}^{1}$. We call $(K / F, \chi)$ an admissible 1-pair if $\chi$ does not factor through $\mathrm{N}_{K / E}$, for any field $E$ with $F \subset E \varsubsetneqq K$. We write $P^{(1)}(F)$ for the set of isomorphism classes of admissible 1-pairs over $F$.

Let $(K / F, \chi) \in P^{(1)}(F)$. We can thus form the endo-class $\mathrm{R}_{K / F} \mathcal{E}_{K}(\chi) \in \mathcal{E}(F)$.

Theorem 1.3. Let $(K / F, \chi) \in P^{(1)}(F)$, and write $\Theta_{\chi}=\mathrm{R}_{K / F} \mathcal{E}_{K}(\chi)$. Then $\Theta_{\chi} \in \mathcal{E}^{\mathrm{et}}(F)$ and $\operatorname{deg} \Theta_{\chi}=[K: F]$. Moreover, the map

$$
\begin{gathered}
P^{(1)}(F) \longrightarrow \mathcal{E}^{\mathrm{et}}(F), \\
(K / F, \chi) \longmapsto \Theta_{\chi}
\end{gathered}
$$

is a canonical bijection, which is natural with respect to automorphisms of the base field $F$. 
Proof. Let $(K / F, \chi) \in P^{(1)}(F)$; by definition, the endo-class $\Theta_{\chi} \in \mathcal{E}(F)$ has a $K / F$ lift of degree 1. Since $K / F$ is tamely ramified, it follows that $\Theta_{\chi}$ is tame and $K / F$ splits $\Theta_{\chi}$. In particular, $\operatorname{deg} \Theta_{\chi}$ divides $[K: F]$. Furthermore, $K$ contains a splitting field $E / F$ for $\Theta_{\chi}$ (Corollary 1.1). There is, therefore, a character $\phi$ of $U_{E}^{1}$ such that $\Theta_{\chi}=\mathrm{R}_{E / F} \mathcal{E}_{E}(\phi)$ and $\mathcal{E}_{K}(\chi)$ is the $K / F$-lift of $\mathcal{E}_{E}(\phi)$. That is, $\chi=\phi \circ \mathrm{N}_{K / E}$; since $(K / F, \chi)$ is admissible, we have $E=K$ and $\operatorname{deg} \Theta_{\chi}=[E: F]=[K: F]$, as required.

For $i=1,2$, let $\left(K_{i} / F, \chi_{i}\right) \in P^{(1)}(F)$, and assume that $\Theta_{\chi_{1}}=\Theta_{\chi_{2}}$. We choose a finite, tamely ramified Galois extension $L / F$ containing both $K_{i}$. We set $\chi_{i}^{L}=$ $\chi_{i} \circ \mathrm{N}_{L / K_{i}} \in \widehat{U}_{L}^{1}$. By hypothesis, we have

$$
\mathrm{R}_{L / F} \mathcal{E}_{L}\left(\chi_{1}^{L}\right)=\mathrm{R}_{L / F} \mathcal{E}_{L}\left(\chi_{2}^{L}\right) .
$$

By [3, $9.13 \mathrm{et} \mathrm{seq}$ ], this implies that the endo-classes $\mathcal{E}_{L}\left(\chi_{i}^{L}\right)$, hence also the characters $\chi_{i}^{L}$ themselves, are conjugate under $\operatorname{Gal}(L / F)$. Since $K_{i} / F$ is the least subextension of $L / F$ such that $\chi_{i}^{L}$ factors through $\mathrm{N}_{L / K_{i}}$ (cf. (A.1), Lemma (3)), this conjugation carries the pair $\left(K_{1} / F, \chi_{1}\right)$ to $\left(K_{2} / F, \chi_{2}\right)$, whence the pairs $\left(K_{i} / F, \chi_{i}\right)$ are conjugate. In other words, $\left(K_{1} / F, \chi_{1}\right) \cong\left(K_{2} / F, \chi_{2}\right)$, and we have shown that the map $P^{(1)}(F) \rightarrow \mathcal{E}^{\text {et }}(F)$ is injective.

Let $\Theta \in \mathcal{E}^{\text {et }}(F)$ have degree $n$. There is a vector space $V$ of dimension $n$ over $F$, a simple stratum $[\mathfrak{A}, l, 0, \beta]$ in $\operatorname{End}_{F}(V)$ and a simple character $\theta \in \mathcal{C}(\mathfrak{A}, \beta)$ with $\Theta=\mathcal{E}_{F}(\theta)$. We put $E=F[\beta]$, so that $[E: F]=n$. We have $U_{E}^{1}=E^{\times} \cap H^{1}(\beta, \mathfrak{A})$, and we form the character $\chi=\theta \mid U_{E}^{1}$. As in $1.2, \chi$ is a simple character over $E$, and (by definition $3,9.4] \mathcal{E}_{E}(\chi)$ is an $E / F$-lift of $\Theta$. We first have to show that $(E / F, \chi)$ is an admissible 1-pair. Suppose we have a subfield $K, F \subset K \subset E$, such that $\chi$ factors through $\mathrm{N}_{E / K}$, say $\chi=\phi \circ \mathrm{N}_{E / K}$, for some $\phi \in \widehat{U}_{K}^{1}$. Then $\mathcal{E}_{K}(\phi)$ is a $K / F$-lift of $\Theta$, of degree 1 . That is, $K / F$ splits $\Theta$, whence $n=\operatorname{deg} \Theta$ divides $[K: F]$. By 1.1 , we have $K=E$ and $(E / F, \chi) \in P^{(1)}(F)$. Therefore $\Theta=\Theta_{\chi}$, and our map is surjective. It is therefore bijective, as required.

The operation of tame lifting of endo-classes is natural with respect to automorphisms of the base field $F$. The same therefore applies to the bijection of the theorem.

Let us exhibit the inverse of the map $P^{(1)}(F) \rightarrow \mathcal{E}^{\text {et }}(F)$, as implied by the proof of the theorem. Suppose $\Theta \in \mathcal{E}^{\text {et }}(F)$ is the endo-class of $\theta \in \mathcal{C}(\mathfrak{A}, \beta)$, for a simple stratum $[\mathfrak{A}, l, 0, \beta]$ in $A=\operatorname{End}_{F}(V)$, for some vector space $V$. Set $E=F[\beta]$ and let $B$ denote the $A$-centralizer of $E$. Thus $\mathfrak{B}=B \cap \mathfrak{A}$ is a hereditary $\mathfrak{o}_{E}$-order in $B$ and we have $H^{1}(\beta, \mathfrak{A}) \cap B^{\times}=U_{\mathfrak{B}}^{1}$. The restriction of $\theta$ to $U_{\mathfrak{B}}^{1}$ is of the form $\phi \circ \operatorname{det}_{B}$, for a uniquely determined character $\phi$ of $U_{E}^{1}$. The image of $\Theta$ in $P^{(1)}(F)$ is then the $F$-isomorphism class of $(E / F, \phi)$.

Remark. The base field restriction map $\mathrm{R}_{K / F}: \mathcal{E}(K) \rightarrow \mathcal{E}(F)$ does not depend on the underlying choice of character $\psi_{F}$ (cf. [6, Remark \$1]). The same, therefore, applies to the map (1.3.1).

1.4. Let $[\mathfrak{A}, l, 0, \beta]$ be a simple stratum in $\mathrm{M}_{n}(F)$, say, and let $\theta \in \mathcal{C}(\mathfrak{A}, \beta)$. Let $\chi$ be a character of $U_{F}^{1}$ and consider the character $\theta^{\prime}=\theta \cdot(\chi \circ \operatorname{det})$ of $H^{1}(\beta, \mathfrak{A})$. There is then a simple stratum $\left[\mathfrak{A}, l^{\prime}, 0, \beta^{\prime}\right]$, where $\beta^{\prime}=\beta+a$, for some $a \in F^{\times}$, such that $H^{1}\left(\beta^{\prime}, \mathfrak{A}\right)=H^{1}(\beta, \mathfrak{A})$ and $\theta^{\prime} \in \mathfrak{C}\left(\mathfrak{A}, \beta^{\prime}\right)$ : see [10, Appendix] for details of this construction. 
In the present context, let $(K / F, \xi) \in P^{(1)}(F)$, and let $\chi$ be a character of $U_{F}^{1}$. Setting $\xi^{\prime}=\xi \cdot\left(\chi \circ \mathrm{N}_{K / F}\right)$, the 1-pair $\left(K / F, \xi^{\prime}\right)$ is admissible, and

$$
\Theta_{\xi^{\prime}}=\mathcal{E}_{F}\left(\theta_{\xi} \cdot(\chi \circ \operatorname{det})\right),
$$

for any simple character $\theta_{\xi}$ such that $\mathcal{E}_{F}\left(\theta_{\xi}\right)=\Theta_{\xi}$.

\section{EsSEntially tame SUPERCUSPIDAL REPRESENTATIONS}

Let $\pi$ be an irreducible supercuspidal representation of $G=\mathrm{GL}_{n}(F)$. As in the introduction, we let $t(\pi)$ denote the number of unramified characters $\chi$ of $F^{\times}$such that $\chi \pi \cong \pi$. Thus $t(\pi)$ is an integer dividing $n$; we say that $\pi$ is essentially tame if $p$ does not divide $n / t(\pi)$. We write $\mathcal{A}_{n}^{\text {et }}(F)$ for the set of equivalence classes of essentially tame, irreducible supercuspidal representations of $\mathrm{GL}_{n}(F)$.

The aim of this section is to construct a canonical bijection $P_{n}(F) \rightarrow \mathcal{A}_{n}^{\text {et }}(F)$, for each $n \geq 1$.

2.1. We recall briefly the main features of the classification [9] of irreducible supercuspidal representations of $G=\mathrm{GL}_{n}(F)$, in terms of maximal simple types. Let $\pi \in \mathcal{A}_{n}^{0}(F)$. According to [9 (8.4.1)], $\pi$ contains a unique $G$-conjugacy class of maximal simple types $(J, \lambda)$, in the sense of $[9,(5.5 .10,6.2)]$. Moreover, if $\pi$ contains the maximal simple type $(J, \lambda)$ and $\mathbf{J}_{\lambda}$ denotes the $G$-normalizer of the pair $(J, \lambda)$, there is a unique representation $\Lambda$ of $\mathbf{J}_{\lambda}$ which occurs in $\pi$ and satisfies $\Lambda \mid J \cong \lambda$. We then have

$$
\pi \cong c-\operatorname{Ind}_{\mathbf{J}_{\lambda}}^{G} \Lambda .
$$

(We refer to pairs of the form $\left(\mathbf{J}_{\lambda}, \Lambda\right)$ as extended maximal simple types.)

The definition loc. cit. divides the maximal simple types into two subclasses. In the first, dubbed "of level zero", we have $J=U_{\mathfrak{A}}$, where $\mathfrak{A} \cong \mathrm{M}_{n}\left(\mathfrak{o}_{F}\right)$. The representation $\lambda$ is the inflation of an irreducible cuspidal representation $\bar{\lambda}$ of $U_{\mathfrak{A}} / U_{\mathfrak{A}}^{1} \cong \mathrm{GL}_{n}\left(\mathbb{k}_{F}\right)$. The group $\mathbf{J}_{\lambda}$ is $F^{\times} J$. A representation $\pi \in \mathcal{A}_{n}^{0}(F)$ is said to be of level zero if it contains a maximal simple type of level zero.

In the second case, there is a simple stratum $[\mathfrak{A}, l, 0, \beta]$ in $A=\mathrm{M}_{n}(F)$, such that $\mathfrak{A}$ is maximal among $F[\beta]^{\times}$-stable hereditary $\mathfrak{o}_{F}$-orders in $A$, and a simple character $\theta \in \mathfrak{C}(\mathfrak{A}, \beta)$ which occurs in $\pi$ : indeed, $\theta$ is the unique simple character occurring in $\pi$, up to $G$-conjugacy. The group $J=J^{0}(\beta, \mathfrak{A})$ is the $U_{\mathfrak{A} \text {-normalizer }}$ of $\theta$; the natural representation $\lambda$ of $J$ on the $\theta$-isotypic space in $\pi$ gives a maximal simple type $(J, \lambda)$ in $\pi$. (We recall more of the representation $\lambda$ below.) The group $\mathbf{J}_{\lambda}$ is $F[\beta]^{\times} J$.

For $\pi \in \mathcal{A}_{n}^{0}(F)$, we define an invariant $\Theta(\pi) \in \mathcal{E}(F)$ as follows: $\Theta(\pi)$ is trivial if $\pi$ is of level zero, while otherwise $\Theta(\pi)=\mathcal{E}_{F}(\theta)$, where $\theta$ is a simple character occurring in $\pi$.

Proposition 2.1. Let $\pi \in \mathcal{A}_{n}^{0}(F)$; then $\pi$ is essentially tame if and only if $\Theta(\pi) \in$ $\mathcal{E}^{\mathrm{et}}(F)$.

Proof. If $\pi$ has level zero, then $t(\pi)=n$ while, if $\pi$ contains a simple character $\theta \in \mathcal{C}(\mathfrak{A}, \beta)$, then $t(\pi)=n / e(F[\beta] \mid F)[9,(6.2 .5)]$. In other words, $n / t(\pi)=e(\Theta(\pi))$, whence the result follows. 
2.2. It is convenient to deal separately with the first subclass. Let $\mathcal{A}_{n}^{0}(F)_{0}$ denote the set of $\pi \in \mathcal{A}_{n}^{0}(F)$ which are of level zero. Let $P_{n}(F)_{0}$ denote the set of $(E / F, \xi) \in P_{n}(F)$ for which $\xi \mid U_{E}^{1}=1$.

Take $(E / F, \xi) \in P_{n}(F)_{0}$. By definition, $E / F$ is unramified. We set $\Sigma=$ $\operatorname{Gal}(E / F)$. The admissibility of the pair $(E / F, \xi)$ is then equivalent to $\xi$ being $\Sigma$-regular, in the sense that the conjugates $\xi^{\sigma}, \sigma \in \Sigma$, are distinct. The character $\xi \mid U_{E}$ is the inflation of a character $\bar{\xi}$ of $\mathbb{k}_{E}^{\times}$. If we identify $\Sigma$ with Gal $\left(\mathbb{k}_{E} / \mathbb{k}_{F}\right)$ by reduction, the admissibility of $(E / F, \xi)$ is again equivalent to $\bar{\xi}$ being $\Sigma$-regular.

We now use the Green parametrization [13: this gives a canonical bijection $\chi \mapsto \bar{\lambda}_{\chi}$ between the set of $\Sigma$-orbits of $\Sigma$-regular characters $\chi$ of $\mathbb{k}_{E}^{\times}$and the set of equivalence classes of irreducible cuspidal representations $\bar{\lambda}$ of $\mathrm{GL}_{n}\left(\mathbb{k}_{F}\right)$.

We choose an $F$-embedding of $E$ in $A=\mathrm{M}_{n}(F)$, and let $\mathfrak{A}$ be the unique $E^{\times}$stable hereditary $\mathfrak{o}_{F}$-order in $A$ : we have $\mathfrak{A} \cong \mathrm{M}_{n}\left(\mathfrak{o}_{F}\right)$ and $U_{\mathfrak{A}} / U_{\mathfrak{A}}^{1} \cong \mathrm{GL}_{n}\left(\mathbb{k}_{F}\right)$. The character $\bar{\xi}$ gives a cuspidal representation $\bar{\lambda}_{\xi}$ of $\mathrm{GL}_{n}\left(\mathbb{k}_{F}\right)$, which we inflate to a representation $\lambda_{\xi}$ of $U_{\mathfrak{A}}$. We extend $\lambda_{\xi}$ to a representation $\Lambda_{\xi}$ of $\mathbf{J}=F^{\times} U_{\mathfrak{A}}$ by deeming that $\Lambda_{\xi} \mid F^{\times}$be a multiple of $\xi$. We set

$$
{ }_{F} \pi_{\xi}=c-\operatorname{Ind}_{\mathbf{J}}^{G} \Lambda_{\xi} .
$$

Proposition 2.2. Let $(E / F, \xi) \in P_{n}(F)_{0}$ and define ${ }_{F} \pi_{\xi}$ as in (2.2.1). Then:

(1) ${ }_{F} \pi_{\xi} \in \mathcal{A}_{n}^{0}(F)_{0}$, and the equivalence class of $\pi$ depends only on that of the pair $(E / F, \xi)$;

(2) the map $(E / F, \xi) \mapsto{ }_{F} \pi_{\xi}$ is a canonical bijection $P_{n}(F)_{0} \rightarrow \mathcal{A}_{n}^{0}(F)_{0}$.

Proof. The first assertion is immediate, so we have a well-defined map $P_{n}(F)_{0} \rightarrow$ $\mathcal{A}_{n}^{0}(F)_{0}$. If we take $\pi \in \mathcal{A}_{n}^{0}(F)_{0}$, it contains a maximal simple type $\left(\mathrm{GL}_{n}\left(\mathfrak{o}_{F}\right), \lambda\right)$ (unique up to conjugacy), where $\lambda$ is inflated from an irreducible cuspidal representation of $\mathrm{GL}_{n}\left(\mathbb{k}_{F}\right)$. We reverse the procedure above to construct from $\pi$ a pair $(E / F, \xi) \in P_{n}(F)_{0}$. This gives a well-defined map $\mathcal{A}_{n}^{0}(F)_{0} \rightarrow P_{n}(F)_{0}$, inverse to the first one.

2.3. We take an admissible pair $(E / F, \xi)$, of degree $n$, such that $\xi \mid U_{E}^{1} \neq 1$. We construct from this pair a representation ${ }_{F} \pi_{\xi} \in \mathcal{A}_{n}^{\text {et }}(F)$, the equivalence class of which will depend only on the isomorphism class of $(E / F, \xi)$.

Let $E^{\prime} / F$ be the minimal sub-extension of $E / F$ such that $\xi \mid U_{E}^{1}$ factors through $\mathrm{N}_{E / E^{\prime}}$ (see Appendix (A.1), Lemma (3)), and write $\xi \mid U_{E}^{1}=\phi \circ \mathrm{N}_{E / E^{\prime}}$. We choose a simple stratum $[\mathfrak{A}, l, 0, \beta]$ in $A=\mathrm{M}_{n}(F)$ and a simple character $\theta \in \mathcal{C}(\mathfrak{A}, \beta)$ such that $\mathcal{E}_{F}(\theta)=\mathrm{R}_{E^{\prime} / F} \mathcal{E}_{E^{\prime}}(\phi)$. We then have $F[\beta] \cong E^{\prime}$; we henceforward identify $E^{\prime}$ with $F[\beta] \subset A$.

We can choose $\mathfrak{A}$ to be maximal among $E^{\prime \times}$-stable hereditary $\mathfrak{o}_{F}$-orders in $A$. That done, the $G=\mathrm{GL}_{n}(F)$-conjugacy class of the pair $(\mathfrak{A}, \theta)$ is determined by the $F$-isomorphism class of $\left(E^{\prime} / F, \phi\right)$ (and hence by that of $\left.(E / F, \xi)\right)$. The pair $(\mathfrak{A}, \theta)$ determines the groups $J^{1}=J^{1}(\beta, \mathfrak{A}), J^{0}=J^{0}(\beta, \mathfrak{A})$ and $\mathbf{J}=E^{\prime \times} J^{0}$ : these groups are the normalizers of $\theta$ in, respectively, $U_{\mathfrak{A}}^{1}, U_{\mathfrak{A}}$ and $G$.

Let $B$ denote the $A$-centralizer of $E^{\prime}$. Let $\eta$ be the unique irreducible representation of $J^{1}$ which contains $\theta[9,(5.1 .1)]$.

Lemma 1. There exists a unique irreducible representation $\kappa$ of $J$ such that

(1) $\kappa \mid J^{1} \cong \eta$; 
(2) $\kappa$ is intertwined by every element of $B^{\times}$;

(3) the character det $\kappa$ has finite, p-power order.

Proof. This follows from [9, (5.2.2)].

To proceed further, we have to choose a prime element $\varpi_{F}$ of $F$.

Lemma 2. There is a unique representation $\widetilde{\kappa}$ of $\mathbf{J}$ satisfying the following conditions:

(1) $\widetilde{\kappa} \mid J \cong \kappa$;

(2) $\varpi_{F} \in \operatorname{Ker} \widetilde{\kappa}$;

(3) $\operatorname{det} \widetilde{\kappa}$ has finite, $p$-power order.

Proof. We have $\mathbf{J}=E^{\times} J=E^{\prime \times} J$ and $E^{\prime \times} \cap J=U_{E^{\prime}}$. We can therefore extend $\kappa$ by triviality to the group $\left\langle\varpi_{F}, J\right\rangle$ generated by $\varpi_{F}$ and $J$. The quotient $\mathbf{J} /\left\langle\varpi_{F}, J\right\rangle$ is cyclic of order $e\left(E^{\prime} \mid F\right)$. Since $p \nmid e\left(E^{\prime} \mid F\right), \kappa$ admits a unique extension $\widetilde{\kappa}$ of the required form.

For the next step, we use the same prime element $\varpi_{F}$ to impose a factorization on the quasicharacter $\xi$.

Lemma 3. There is a unique character $\xi_{\mathrm{w}}$ of $E^{\times}$with the following properties:

(1) $\xi_{\mathrm{w}}\left|U_{E}^{1}=\xi\right| U_{E}^{1}$;

(2) $\xi_{\mathrm{w}}\left(\varpi_{F}\right)=1$;

(3) $\xi_{\mathrm{w}}$ has finite, p-power order.

The proof is immediate. We now set $\xi_{\mathrm{t}}=\xi_{\mathrm{w}}^{-1} \xi$. The quasicharacter $\xi_{\mathrm{t}}$ is tamely ramified, and the pair $\left(E / E^{\prime}, \xi_{\mathrm{t}}\right)$ is admissible.

Let $\mathfrak{B}=\mathfrak{A} \cap B$. Thus $\mathfrak{B}$ is a maximal $\mathfrak{o}_{E^{\prime}}$-order in $B$. We have $J=U_{\mathfrak{B}} J^{1}$ and $J / J^{1} \cong U_{\mathfrak{B}} / U_{\mathfrak{B}}^{1} \cong \mathrm{GL}_{d}\left(\mathbb{k}_{E^{\prime}}\right)$, where $d=\left[E: E^{\prime}\right]$. We follow the procedure of 2.2 to define, from the admissible pair $\left(E / E^{\prime}, \xi_{\mathrm{t}}\right)$, an irreducible representation $\Lambda_{\mathrm{t}}$ of $E^{\times} U_{\mathfrak{B}} / U_{\mathfrak{B}}^{1} \cong \mathbf{J} / J^{1}$. We view $\Lambda_{\mathrm{t}}$ as a representation of $\mathbf{J}$ trivial on $J^{1}$. We put $\Lambda_{\xi}=\Lambda_{\mathrm{t}} \otimes \widetilde{\kappa}$, and note that this definition is independent of the intermediate choice of $\varpi_{F}$.

At this point, it becomes useful to condense our notation a little:

Notation. Let $(E / F, \xi) \in P_{n}(F)$, and let $E^{\prime} / F$ be the minimal sub-extension of $E / F$ such that $\xi \mid U_{E}^{1}$ factors through $\mathrm{N}_{E / E^{\prime}}$. Thus $\xi \mid U_{E}^{1}=\xi^{\prime} \circ \mathrm{N}_{E / E^{\prime}}$, for a uniquely determined character $\xi^{\prime}$ of $U_{E^{\prime}}^{1}$. We put

$$
\Theta_{\xi}=\Theta_{\xi^{\prime}}=\mathrm{R}_{E^{\prime} / F}\left(\mathcal{E}_{E^{\prime}}\left(\xi^{\prime}\right)\right)=\mathrm{R}_{E / F}\left(\varepsilon_{E}\left(\xi \mid U_{E}^{1}\right)\right) .
$$

Proposition 2.3. The representation ${ }_{F} \pi_{\xi}=c-\operatorname{Ind}_{\mathbf{J}}^{G} \Lambda_{\xi}$ is irreducible, supercuspidal, essentially tame, and it satisfies $\Theta\left({ }_{F} \pi_{\xi}\right)=\Theta_{\xi}$. The equivalence class of ${ }_{F} \pi_{\xi}$ depends only on the isomorphism class of the pair $(E / F, \xi)$.

Proof. Setting $\lambda=\Lambda_{\xi} \mid J$, the pair $(J, \lambda)$ is a maximal simple type in $G$ and $\left(\mathbf{J}, \Lambda_{\xi}\right)$ is an extended maximal simple type. Thus $\pi={ }_{F} \pi_{\xi}$ lies in $\mathcal{A}_{n}^{0}(F)$ and by construction, $\Theta(\pi)=\Theta_{\xi} \in \mathcal{E}^{\text {et }}(F)$. Thus $\pi$ is essentially tame. By Theorem 1.3 and the uniqueness of the pair $(\mathfrak{A}, \theta)$ described above, different choices of pairs $(E / F, \xi)$ in the same isomorphism class lead to conjugate types $(\mathbf{J}, \Lambda)$ and hence equivalent representations ${ }_{F} \pi_{\xi}$. 
Taking Proposition 2.2 into account, the operation $(E / F, \xi) \mapsto{ }_{F} \pi_{\xi}$ gives a welldefined map $P_{n}(F) \rightarrow \mathcal{A}_{n}^{\text {et }}(F)$.

Theorem 2.3. The map

$$
\begin{gathered}
P_{n}(F) \longrightarrow \mathcal{A}_{n}^{\text {et }}(F), \\
(E / F, \xi) \longmapsto{ }_{F} \pi_{\xi}
\end{gathered}
$$

is a bijection.

Proof. A representation $\pi \in \mathcal{A}_{n}^{\text {et }}(F)$ contains an extended maximal simple type $(\mathbf{J}, \Lambda)$, of level zero or based on a simple stratum [9, (8.4.1)]. In the latter case, the stratum is tamely ramified by Proposition 2.1. Any such $(\mathbf{J}, \Lambda)$ arises from an element of $P_{n}(F)$ as above: all steps in the construction are reversible. Thus the map (2.3.1) is surjective.

For $i=1,2$, let $\left(E_{i} / F, \xi_{i}\right) \in P_{n}(F)$ and suppose that ${ }_{F} \pi_{\xi_{1}}={ }_{F} \pi_{\xi_{2}}=\pi$, say. Let $E_{i}^{\prime} / F$ be the minimal sub-extension of $E_{i} / F$ such that $\xi_{i} \mid U_{E_{i}}^{1}$ factors through $\mathrm{N}_{E_{i} / E_{i}^{\prime}}$. Let $\phi_{i}$ be the character of $U_{E_{i}^{\prime}}^{1}$ such that $\xi_{i} \mid U_{E_{i}}^{1}=\phi_{i} \circ \mathrm{N}_{E_{i} / E_{i}^{\prime}}$. By Theorem 1.3 and the proposition, the admissible 1-pairs $\left(E_{i}^{\prime} / F, \phi_{i}\right)$ are isomorphic, so we may take $E_{1}^{\prime}=E_{2}^{\prime}=E^{\prime}$ and $\phi_{1}=\phi_{2}$. The extensions $E_{i} / E^{\prime}$ are unramified of the same degree, so we may as well assume $E_{1}=E_{2}=E$, say, and $\xi_{1}\left|U_{E}^{1}=\xi_{2}\right| U_{E}^{1}$.

If we follow through the constructions above, for the pairs $\left(E / F, \xi_{i}\right)$, we arrive at the same stratum $[\mathfrak{A}, l, 0, \beta]$ and the same $\theta \in \mathfrak{C}(\mathfrak{A}, \beta)$. Furthermore, if we choose a prime element $\varpi_{F}$ of $F$ to factorize the $\xi_{i}$ as in Lemma 3 , we get $\xi_{1, \mathrm{w}}=\xi_{2, \mathrm{w}}$. The corresponding extended simple types $\left(\mathbf{J}, \Lambda_{i}\right), \mathbf{J}=E^{\prime \times} J^{0}(\beta, \mathfrak{A})$, lie in the same representation $\pi$ and so are $G$-conjugate. Indeed, they are conjugate under the $G$-normalizer of $\theta$, which is none other than $\mathbf{J}$. In other words, $\Lambda_{1}=\Lambda_{2}$. In the factorizations $\Lambda_{i}=\Lambda_{i, \mathrm{t}} \otimes \widetilde{\kappa}$, the factors $\Lambda_{i, \mathrm{t}}$ are equivalent, so the admissible pairs $\left(E / E^{\prime}, \xi_{i} \xi_{i, \mathrm{w}}^{-1}\right)$ are $E^{\prime}$-isomorphic. We conclude that the pairs $\left(E / F, \xi_{i}\right)$ are $F$-isomorphic, as required.

Remark 1. The only debatable step in the construction of ${ }_{F} \pi_{\xi}$ is the definition of the factor $\widetilde{\kappa}$ held to correspond to the wildly ramified factor $\xi_{\mathrm{w}}$ of $\xi$. Other choices of $\widetilde{\kappa}$ are possible, and some give variants of (2.3.1) with smoother properties. We shall analyze this phenomenon elsewhere. For the present, the version given above has the advantage of being simple, straightforward, and in need of no preliminary analysis.

Remark 2. Constructions such as (2.3.1) originated in [18], and were further developed in 23]. Slightly different conventions are used in [24, where the relation between the approaches is discussed. Our method is actually identical to that of 24, as one verifies easily, starting from Theorem 1.3. We have subsumed the extensive details of the earlier papers into the general theory of [9] and [3], to obtain the simple and direct proof of bijectivity. This option was not available to those authors who were also confined to the case $p \nmid n$ and $F$ of characteristic zero.

2.4. We collect some simple properties of the bijection (2.3.1).

Proposition 2.4. (1) The map (2.3.1) is natural with respect to automorphisms of the base field $F$.

(2) If $(K / F, \xi) \in P_{n}(F)$ and $\pi={ }_{F} \pi_{\xi}$, then

$$
{ }_{F} \pi_{\xi^{-1}}=\check{\pi} \quad \text { and } \quad \omega_{\pi}=\xi \mid F^{\times} .
$$


(3) Let $(K / F, \xi) \in P_{n}(F)$ and let $\chi$ be a quasicharacter of $F^{\times}$. Write $\chi_{K}=$ $\chi \circ \mathrm{N}_{K / F}$. Then $\left(K / F, \xi \cdot \chi_{K}\right) \in P_{n}(F)$ and

$$
{ }_{F} \pi_{\xi \cdot \chi_{K}}=\chi \cdot{ }_{F} \pi_{\xi}
$$

(4) The map (2.3.1) is natural with respect to (not necessarily continuous) automorphisms of $\mathbb{C}$, that is,

$$
{ }_{F} \pi_{\gamma \circ \xi}=\gamma \circ{ }_{F} \pi_{\xi},
$$

for $(E / F, \xi) \in P_{n}(F)$ and $\gamma \in$ Aut $\mathbb{C}$.

All of these assertions are proved by following through the construction of ${ }_{F} \pi_{\xi}$, step by step and, for (3), comparing with 1.4.

\section{The Langlands CORRESPONDENCE AND the NaÏVE CORRESPONDENCE}

We make an initial comparison between the constructions of $\S 2$ and the Langlands correspondence for essentially tame representations.

3.1. We recall that we have a canonical bijection $P_{n}(F) \rightarrow \mathcal{G}_{n}^{\text {et }}(F)$ given by $(E / F, \xi)$ $\mapsto \operatorname{Ind}_{E / F} \xi$ (A.3). Combining this with Theorem 2.3, we have the first statement of:

Theorem 3.1. For each $n \geq 1$, the maps

$$
\begin{aligned}
& P_{n}(F) \longrightarrow \mathcal{G}_{n}^{\text {et }}(F), \quad P_{n}(F) \longrightarrow \mathcal{A}_{n}^{\text {et }}(F), \\
& (E / F, \xi) \longmapsto \operatorname{Ind}_{E / F} \xi, \quad(E / F, \xi) \longmapsto{ }_{F} \pi_{\xi}
\end{aligned}
$$

together induce a bijection

$$
\mathcal{N}={ }_{F} \mathcal{N}_{n}: \mathcal{G}_{n}^{\text {et }}(F) \stackrel{\approx}{\rightarrow} \mathcal{A}_{n}^{\text {et }}(F) .
$$

The map $\mathcal{N}$ has the following properties:

(1) ${ }_{F} \mathcal{N}_{1}$ is the bijection $\mathcal{G}_{1}(F) \rightarrow \mathcal{A}_{1}(F)$ of local class field theory;

(2) ${ }_{F} \mathcal{N}_{n}$ is natural with respect to automorphisms of the base field $F$;

(3) if $\sigma \in \mathcal{G}_{n}^{\mathrm{et}}(F)$ and $\pi=\mathcal{N}(\sigma)$, then $\mathcal{N}(\check{\sigma})=\check{\pi}$;

(4) if $\sigma \in \mathcal{G}_{n}^{\text {et }}(F)$ and $\chi \in \mathcal{G}_{1}(F)$, then $\mathcal{N}(\chi \otimes \sigma)=\chi \cdot \mathcal{N}(\sigma)$;

(5) if $\sigma=\operatorname{Ind}_{E / F} \xi$, for $(E / F, \xi) \in P_{n}(F)$, and $\pi=\mathcal{N}(\sigma)={ }_{F} \pi_{\xi}$, then

$$
\operatorname{det} \sigma=\omega_{\pi} \delta_{E / F} \text {. }
$$

Proof. Property (1) is a matter of definition, while the others follow from Proposition 2.4.

Remark. We observe also that $\mathcal{N}$ commutes with the natural actions of Aut $\mathbb{C}$.

3.2. Let ${ }_{F} \mathcal{L}_{n}: \mathcal{G}_{n}^{0}(F) \rightarrow \mathcal{A}_{n}^{0}(F)$ be the Langlands correspondence. We have observed that ${ }_{F} \mathcal{L}_{n}$ satisfies the analogue of Theorem 3.1(4), so it induces a bijection

$$
{ }_{F} \mathcal{L}_{n}^{\mathrm{et}}: \mathcal{G}_{n}^{\mathrm{et}}(F) \stackrel{\approx}{\rightarrow} \mathcal{A}_{n}^{\mathrm{et}}(F) .
$$

We usually write simply ${ }_{F} \mathcal{L}_{n}^{\text {et }}=\mathcal{L}$.

Let $K / F$ be a cyclic field extension of degree $d$. We then have the operations of base change $\mathrm{b}_{K / F}$ [1], [17], and automorphic induction $\mathrm{A}_{K / F}$ [16], [17]. These correspond, via the Langlands correspondence, to the operations of restriction and induction connecting representations of the Weil-Deligne groups of $F$ and $K l o c$. cit. Translating this in terms of our constructions, we have: 
Proposition 3.2. The Langlands correspondence induces a bijection

$$
\mathcal{L}={ }_{F} \mathcal{L}_{n}^{\text {et }}: \mathcal{G}_{n}^{\mathrm{et}}(F) \longrightarrow \mathcal{A}_{n}^{\mathrm{et}}(F),
$$

for each $n \geq 1$. This map satisfies the analogues of (1)-(4) of Theorem 3.1, while instead of (5) we have

$$
\omega_{\pi}=\operatorname{det} \sigma, \quad \pi=\mathcal{L}(\sigma) .
$$

In addition:

(6) Let $(E / F, \xi) \in P_{n}(F)$ and let $K / F$ be a cyclic sub-extension of $E / F$. Put $\tau=\operatorname{Ind}_{E / K} \xi, \rho=\mathcal{L}(\tau), \sigma=\operatorname{Ind}_{E / F} \xi$ and $\pi=\mathcal{L}(\sigma)$. Then $\pi$ is automorphically induced by $\rho$ :

$$
\pi=\mathrm{A}_{K / F} \rho
$$

(7) Let $(E / F, \xi) \in P_{n}(F)$, let $L / F$ be a finite, cyclic tamely ramified extension, and suppose that the pair $\left(E L / L, \xi_{L}\right)$ is admissible, where $\xi_{L}=\xi \circ \mathrm{N}_{E L / E}$. Set $\sigma=\operatorname{Ind}_{E / F} \xi, \pi=\mathcal{L}(\sigma)$ and $\sigma_{L}=\operatorname{Ind}_{E L / L} \xi_{L}=\sigma \mid \mathcal{W}_{L}$. Then

$$
\mathcal{L}\left(\sigma_{L}\right)=\mathrm{b}_{L / F} \pi \text {. }
$$

We recall [6, (7.1)] that in (6) we have $\Theta(\pi)=\mathrm{R}_{K / F} \Theta(\rho)$ while, in (7), $\Theta\left(\mathrm{b}_{L / F} \pi\right)$ is the unique $L / F$-lift of $\Theta(\pi)$.

Remark. The list of properties in the proposition is sufficient to determine the family ${ }_{F} \mathcal{L}_{n}^{\text {et }}$ uniquely. This is not a new observation: it is implicit in [22] and also in our arguments. We sketch a direct proof in 3.5 below.

3.3. We now give our main results, using the notation of 2.3 .

Theorem 3.3. Let $(E / F, \xi) \in P_{n}(F)$; set $\sigma=\operatorname{Ind}_{E / F} \xi \in \mathcal{G}_{n}^{\text {et }}(F)$ and $\pi=\mathcal{L}(\sigma) \in$ $\mathcal{A}_{n}^{\text {et }}(F)$. Then

$$
\Theta(\pi)=\Theta_{\xi}=\Theta\left({ }_{F} \pi_{\xi}\right) .
$$

Proof. Set $\pi^{\prime}=\mathcal{N}(\sigma)={ }_{F} \pi_{\xi}$ and abbreviate $\mathrm{R}_{E / F}\left(\xi \mid U_{E}^{1}\right)=\Theta_{\xi}$. The relation $\Theta_{\xi}=\Theta\left(\pi^{\prime}\right)$ is then given by Propositions 2.2, 2.3.

To prove that $\Theta(\pi)=\Theta_{\xi}$, we first suppose that $E / F$ is totally ramified. Thus $\sigma$ is totally ramified, in the sense that $t(\sigma)=1$. Let $L / F$ be the unramified extension of $F$ generated by a primitive $n$-th root of unity and set $\xi_{L}=\xi \circ \mathrm{N}_{L E / E}$. The pair $\left(E L / L, \xi_{L}\right)$ is then admissible, and we put

$$
\sigma_{L}=\operatorname{Ind}_{E L / L} \xi_{L}=\sigma \mid \mathcal{W}_{L} \in \mathcal{G}_{n}^{\text {et }}(L) .
$$

Set $\rho={ }_{L} \mathcal{L}_{n}\left(\sigma_{L}\right)$; since the extension $E L / L$ is cyclic, we have $\rho=\mathrm{A}_{E L / L} \xi_{L}$. Since $L / F$ is cyclic, we also have $\rho=\mathrm{b}_{L / F} \pi$. We now use [6, (7.1)]. The relation $\rho=\mathrm{A}_{E L / L} \xi_{L}$ implies $\Theta(\rho)=\mathrm{R}_{E L / L} \mathcal{E}_{E L}\left(\xi_{L} \mid U_{E L}^{1}\right)$, while $\rho=\mathrm{b}_{L / F} \pi$ implies $\mathrm{R}_{L / F} \Theta(\rho)=\Theta(\pi)$. In all,

$$
\Theta(\pi)=\mathrm{R}_{L / F} \Theta(\rho)=\mathrm{R}_{E L / F} \mathcal{E}_{E L}\left(\xi_{L} \mid U_{E L}^{1}\right)=\mathrm{R}_{E / F}\left(\xi \mid U_{E}^{1}\right)=\Theta_{\xi},
$$

since the map $\mathrm{R}$ is transitive with respect to the base field extension. This proves the theorem in the case where $E / F$ is totally ramified.

In the general case, let $K / F$ be the maximal unramified sub-extension of $E / F$, put $\tau=\operatorname{Ind}_{E / K} \xi$ and $\rho=\mathcal{L}(\tau)$. Thus $\pi=\mathrm{A}_{K / F} \rho$. By the first case, we have $\Theta(\rho)=\mathrm{R}_{E / K} \mathcal{E}_{E}\left(\xi \mid U_{E}^{1}\right)$, whence loc. cit.

$$
\Theta(\pi)=\mathrm{R}_{K / F} \mathrm{R}_{E / K} \mathcal{E}_{E}\left(\xi \mid U_{E}^{1}\right)=\Theta_{\xi}
$$

This completes the proof of the theorem. 
We can re-interpret the theorem as follows:

Corollary 3.3. Let $(E / F, \xi) \in P_{n}(F)$ and set $\sigma=\operatorname{Ind}_{E / F} \xi$. There exists a tamely ramified character $\mu={ }_{F} \mu_{\xi}$ of $E^{\times}$such that $(E / F, \mu \xi)$ is admissible and

$$
{ }_{F} \mathcal{L}_{n}^{\text {et }}(\sigma)={ }_{F} \pi_{\mu \xi} \text {. }
$$

The character ${ }_{F} \mu_{\xi}$ further satisfies ${ }_{F} \mu_{\xi} \mid F^{\times}=\delta_{E / F}$.

Proof. Let us write $\pi=\mathcal{N}(\sigma)={ }_{F} \pi_{\xi}$ and $\pi^{\prime}=\mathcal{L}(\sigma)$. By Theorem 2.3, there is an admissible pair $\left(E^{\prime} / F, \xi^{\prime}\right) \in P_{n}(F)$ such that $\pi^{\prime}={ }_{F} \pi_{\xi^{\prime}}$. By the theorem, we have

$$
\Theta_{\xi^{\prime}}=\Theta\left(\pi^{\prime}\right)=\Theta_{\xi}=\Theta(\pi) .
$$

Let $K / F$ be the minimal sub-extension of $E / F$ such that $\xi \mid U_{E}^{1}$ factors through $\mathrm{N}_{E / K}$, and let $\phi$ be the character of $U_{K}^{1}$ for which $\xi \mid U_{E}^{1}=\phi \circ \mathrm{N}_{E / K}$. Similarly define $K^{\prime} / F$ and $\phi^{\prime}$. The 1-pairs $(K / F, \phi),\left(K^{\prime} / F, \phi^{\prime}\right)$ are admissible; we have

$$
\mathrm{R}_{K / F} \mathcal{E}_{K}(\phi)=\Theta_{\xi}=\Theta_{\xi^{\prime}}=\mathrm{R}_{K^{\prime} / F} \mathcal{E}_{K^{\prime}}\left(\phi^{\prime}\right),
$$

so $\left(K^{\prime} / F, \phi^{\prime}\right)$ is $F$-isomorphic to $(K / F, \phi)$ (Theorem 1.3). We may as well, therefore, take $K=K^{\prime}$ and $\phi=\phi^{\prime}$. The extensions $E / K, E^{\prime} / K$ are unramified of the same degree $n /[K: F]$, so we are justified in setting $E^{\prime}=E$. These identifications give $\xi\left|U_{E}^{1}=\xi^{\prime}\right| U_{E}^{1}$, so $\xi^{-1} \xi^{\prime}=\mu$ is tamely ramified, as required. The final assertion follows from comparison of (3.2.1) with (3.1.1).

Remark. One can say little about the character ${ }_{F} \mu_{\xi}$ without further analysis. One sees easily that it has finite order: we show elsewhere that it depends only on $\xi \mid U_{E}^{1}$ and that its order divides 4 . Also, we have noted that the correspondence $\mathcal{N}$ is algebraic, in the sense of commuting with Aut $\mathbb{C}$. However, $\mathcal{L}$ is not algebraic, as is clear from the discussion in, for example, [5, §7]. The function $(E / F, \xi) \mapsto{ }_{F} \mu_{\xi}$ therefore cannot be algebraic.

3.4. It will be useful to record a variation on the theme of the corollary. For this, we take an admissible pair $(E / F, \xi) \in P_{n}(F)$ and a cyclic sub-extension $K / F$ of $E / F$, of degree $d$. The pair $(E / K, \xi)$ is then admissible, $(E / K, \xi) \in P_{m}(F)$, where $m d=n$, so we can form the representation $\rho={ }_{K} \pi_{\xi} \in \mathcal{A}_{m}^{\text {et }}(K)$.

Proposition 3.4. Let $\pi=\mathrm{A}_{K / F} \rho$ be the representation of $\mathrm{GL}_{n}(F)$ automorphically induced by $\rho$. The representation $\pi$ is supercuspidal, essentially tame, and there exists a tamely ramified character $\mu={ }_{K / F} \mu_{\xi}$ of $E^{\times}$such that $\pi={ }_{F} \pi_{\mu \xi}$. The character $\mu$ also satisfies $\mu \mid F^{\times}=\delta_{K / F}^{m}$.

Proof. Let $\Sigma=\operatorname{Gal}(K / F)$; we view $E$ as a subfield of a separable algebraic closure $\bar{F} / F$ and extend each $\sigma \in \Sigma$ to an $F$-embedding, also denoted $\sigma$, of $E$ in $\bar{F}$. The admissible pairs $\left(E^{\sigma} / K, \xi^{\sigma}\right), \sigma \in \Sigma$, are then mutually non-isomorphic over $K$. The construction ( $c f$. Proposition 2.4 (1)) yields ${ }_{K} \pi_{\xi^{\sigma}}=\rho^{\sigma}$. The representations $\rho^{\sigma}, \sigma \in \Sigma$, are therefore mutually inequivalent or, in other words, $\rho$ is $\Sigma$-regular. It follows [8, 2.6] that $\pi=\mathrm{A}_{K / F} \rho$ is supercuspidal and [6] (7.1)] $\Theta(\pi)=\mathrm{R}_{K / F} \Theta(\rho)$. Thus $\pi$ is essentially tame, and of the form ${ }_{F} \pi_{\xi^{\prime}}$, for some $\left(E^{\prime} / F, \xi^{\prime}\right) \in P_{n}(F)$.

Furthermore, we have $\Theta(\pi)=\mathrm{R}_{E^{\prime} / F} \mathcal{E}_{E^{\prime}}\left(\xi^{\prime} \mid U_{E^{\prime}}^{1}\right)=\mathrm{R}_{E / F} \mathcal{E}_{E}\left(\xi \mid U_{E}^{1}\right)$. The proof concludes in the same manner as that of Corollary 3.3. 
3.5. We remarked in 3.2 that the family of correspondences ${ }_{F} \mathcal{L}_{n}^{\text {et }}$ is uniquely determined by the properties listed in Proposition 3.2. We shall never use this fact directly, but we give an outline of the proof since it illuminates our strategy.

We take $\sigma \in \mathcal{G}_{n}^{\text {et }}(F)$; we have to construct $\mathcal{L}(\sigma)$ using only properties from the list in section 3.2. We proceed by induction on $n$. If $n=1$, then $\mathcal{L}(\sigma)$ is given by class field theory (the analogue for $\mathcal{L}$ of Theorem 3.1(1)). We therefore assume $n>1$ and we write $\sigma=\operatorname{Ind}_{E / F} \xi$, for some $(E / F, \xi) \in P_{n}(F)$. Suppose first that $E / F$ admits a non-trivial cyclic sub-extension $K / F$. Setting $\tau=\operatorname{Ind}_{E / K} \xi$, the representation $\rho=\mathcal{L}(\tau)$ is known, by the induction hypothesis, while $\mathcal{L}(\sigma)=$ $\mathrm{A}_{K / F} \rho$, by Proposition 3.2(6).

We are so reduced to the case where $E / F$ admits no non-trivial cyclic subextension. Thus $E / F$ is totally ramified and $n=[E: F]$ is odd. Let $\ell$ be the least prime divisor of $n$, and let $L / F$ be the unramified extension generated by a primitive $\ell$-th root of unity. Setting $\xi_{L}=\xi \circ \mathrm{N}_{E L / E}$, the pair $\left(E L / L, \xi_{L}\right)$ is admissible; let $\sigma_{L}=\operatorname{Ind}_{E L / L} \xi_{L}=\sigma \mid \mathcal{W}_{L}$. The extension $E L / L$ surely admits a non-trivial cyclic sub-extension so, by the first case, $\pi_{L}=\mathcal{L}\left(\sigma_{L}\right)$ has been constructed. Setting $\pi=$ $\mathcal{L}(\sigma)$, we have $\pi_{L}=\mathrm{b}_{L / F} \pi$, by Proposition 3.2(7). Let $k=[L: F]$, and let $X_{k}$ be the group of unramified characters $\chi$ of $F^{\times}$satisfying $\chi^{k}=1$. The set of $\rho \in \mathcal{A}_{n}^{\text {et }}(F)$ such that $\mathrm{b}_{L / F} \rho=\pi_{L}$ forms a single $X_{k}$-orbit. The central quasicharacter of $\chi \rho$ is $\chi^{n} \omega_{\rho}$. Since $\omega_{\pi}=\operatorname{det} \sigma(3.2 .1)$ and $(k, n)=1$, the representation $\pi$ is uniquely determined by the two conditions $\mathrm{b}_{L / F} \pi=\pi_{L}$ and $\omega_{\pi}=\operatorname{det} \sigma$.

\section{Totally RAMified REPRESEntations}

We make a preliminary calculation of the character ${ }_{K / F} \mu_{\xi}$ of Proposition 3.4 in a special case. We then specialize further, and prove Theorem B of the introduction.

4.1. Let $(E / F, \xi) \in P_{n}(F)$, and suppose that $E / F$ is totally ramified. In particular, $p \nmid n$. For the moment, we set $\pi={ }_{F} \pi_{\xi}$. Thus $\pi$ is totally ramified in that $t(\pi)=1$.

We choose an $F$-embedding of $E$ in $A=\mathrm{M}_{n}(F)$, and henceforward regard $E$ as a subfield of $A$. As in section 2.3, the pair $(E / F, \xi)$ gives a simple stratum $[\mathfrak{A}, l, 0, \beta]$ in $A$ with $E=F[\beta]$, and a simple character $\theta \in \mathcal{C}(\mathfrak{A}, \beta)$ such that $\theta(x)=\xi(x)$, for $x \in U_{E}^{1}$. We abbreviate $H^{1}=H^{1}(\beta, \mathfrak{A}), J^{1}=J^{1}(\beta, \mathfrak{A})$ and $\mathbf{J}=E^{\times} J^{1}$. We let $\eta$ be the unique irreducible representation of $J^{1}$ which contains $\theta$.

We consider the finite $p$-group $\mathcal{Q}=J^{1} / \operatorname{Ker} \theta$. The centre of $\mathcal{Q}$ is the cyclic group $Z=H^{1} / \operatorname{Ker} \theta$, and $\mathcal{Q} / Z$ is an elementary abelian $p$-group. Since $\theta$ is stable under conjugation by $E^{\times}$, the group $\mathcal{Q}$ inherits an action of $E^{\times}$, the subgroup $F^{\times} U_{E}^{1}$ acting trivially. We therefore view $\mathcal{Q}$ as a module over the finite group $\Gamma=E^{\times} / F^{\times} U_{E}^{1}$; this action of $\Gamma$ stabilizes $\eta$, viewed as a representation of $Q$. Since $E / F$ is totally ramified, the group $\Gamma$ is cyclic.

The group $Q^{\Gamma}$ of $\Gamma$-fixed points is exactly $z$ [4, 4.1, Lemma 1]. We apply the machinery of the Glauberman correspondence [12] (or see [4, Appendix] for a discussion in the present context).

(4.1.1). (1) There exists a unique representation $\tilde{\eta}$ of the group $\Gamma \ltimes \mathcal{Q}$ such that $\tilde{\eta} \mid \mathrm{Q} \cong \eta$ and $\operatorname{det} \tilde{\eta} \mid \Gamma=1$.

(2) There exists a constant $\epsilon_{F}= \pm 1$ such that $\operatorname{tr} \tilde{\eta}(\gamma u)=\epsilon_{F} \xi(u)$, for every generator $\gamma$ of $\Gamma$ and every $u \in U_{E}^{1}$. 
We recall that there is a unique irreducible representation $\Lambda$ of $\mathbf{J}$ such that $\Lambda \mid H^{1}$ is a multiple of $\theta$ and $\pi=c$-Ind $\Lambda$. The construction of $\Lambda$ in section 2.3 can now be expressed more simply in this case, as follows:

Lemma 4.1. Defining $\epsilon_{F}$ as in (4.1.1), the representation $\Lambda$ satisfies

$$
\operatorname{tr} \Lambda(h)=\epsilon_{F} \xi(h),
$$

for every $h \in E^{\times}$such that $v_{E}(h)$ is relatively prime to $n$. This relation characterizes $\Lambda$ among representations of $\mathbf{J}$ which extend $\eta$.

Proof. Take the representation $\tilde{\eta}$ of (4.1.1) and inflate it to a representation $\Lambda_{0}$ of $E^{\times} \ltimes \mathcal{Q}$. Let $\tilde{\xi}$ denote the inflation of $\xi$ to a character of $E^{\times} \ltimes \mathcal{Q}$ and form the representation $\Lambda_{1}=\tilde{\xi} \otimes \Lambda_{0}$ of $E^{\times} \ltimes \mathcal{Q}$. We inflate $\Lambda_{1}$ to a representation $\tilde{\Lambda}_{1}$ of $E^{\times} \ltimes J^{1}$.

There is a canonical surjection $E^{\times} \ltimes J^{1} \rightarrow \mathbf{J}$ given by $(x, j) \mapsto x j$. The kernel is the group of elements $\left(x, x^{-1}\right), x \in U_{E}^{1}$. Since $\tilde{\Lambda}_{1}$ is trivial on this kernel, it is the inflation of an irreducible representation $\Lambda_{2}$ of $\mathbf{J}$. The representation $\Lambda_{2}$ satisfies the character relation of the lemma.

The representation $\Lambda_{2}$ agrees with $\Lambda$ on $F^{\times} J^{1}$, so $\Lambda_{2}=\chi \otimes \Lambda$, for a character $\chi$ of $\mathbf{J} / F^{\times} J^{1} \cong E^{\times} / F^{\times} U_{E}^{1}$. Comparing the constructions, we have, for $y \in E^{\times}$,

$$
\operatorname{det} \Lambda_{2}(y)=\xi(y)^{\operatorname{dim} \eta}=\zeta_{y} \operatorname{det} \Lambda(y)
$$

where $\zeta_{y}$ is some root of unity of $p$-power order. Since $\operatorname{dim} \eta$ is a power of $p$ and $\chi$ has order prime to $p$, we conclude that $\chi=1$, as required.

We consider elements $h$ of $G=\mathrm{GL}_{n}(F)$ such that $v_{F}(\operatorname{det} h)$ is relatively prime to $n$. We call such elements $G$-special.

We will only be concerned with such elements $h$ for which there exists a hereditary $\mathfrak{o}_{F}$-order $\mathfrak{A}$ such that $h \mathfrak{A} h^{-1}=\mathfrak{A}$. Under these conditions, the algebra $F[h]$ is a field, totally ramified and of degree $n$ over $F$.

Proposition 4.1. Let $(E / F, \xi) \in P_{n}(F)$. Suppose that $E / F$ is totally ramified and put $\pi={ }_{F} \pi_{\xi}$.

(1) Let $h \in G$ be $G$-special and suppose that $\operatorname{tr} \pi(h) \neq 0$. Then $h$ is $G$-conjugate to an element of $E^{\times}$.

(2) Let $h \in E^{\times}$be $G$-special. Then

$$
\operatorname{tr} \pi(h)=\epsilon_{F} \sum_{\alpha \in \operatorname{Aut}(E \mid F)} \xi^{\alpha}(h) .
$$

Proof. The condition $\operatorname{tr} \pi(h) \neq 0$ implies that $h$ has a $G$-conjugate in $\mathbf{J}$, so $h$ is an elliptic regular element of $G$. We can therefore apply the Mackey formula [3. (A.14)]

$$
\operatorname{tr} \pi(h)=\sum_{x \in G / \mathbf{J}} \operatorname{tr} \Lambda\left(x^{-1} h x\right),
$$

where we view $\operatorname{tr} \Lambda$ as a function on $G$ vanishing outside $\mathbf{J}$. Clearly, if $\operatorname{tr} \pi(h) \neq 0$, then $h$ is $G$-conjugate to an element of $\mathbf{J}$. We may therefore take $h=b j$, where $j \in J^{1}$ and $b \in E^{\times}$satisfies $v_{E}(b)=v_{F}(\operatorname{det} b)=v_{F}(\operatorname{det} h)$. In particular, $v_{E}(b)$ is relatively prime to $n$. The argument of [3] 15.19] now applies to show that $b j$ is $J^{1}$-conjugate to an element $b j^{\prime}$, where $j^{\prime} \in J^{1}$ commutes with $b$. Since $F[b]=E$, this gives $j^{\prime} \in U_{E}^{1}=E^{\times} \cap J^{1}$, and the first assertion is proved. 
We now take a $G$-special element $h$ of $E^{\times}$and an element $x \in G$ such that $x^{-1} h x \in \mathbf{J}$. As in the first paragraph of the proof, there exists $j \in J^{1}$ such that $h^{\prime}=j^{-1} x^{-1} h x j \in E^{\times}$. We have $E=F\left[h^{\prime}\right]=F[h]$, so conjugation by $x j$ induces an $F$-automorphism of the field $E$ or, equivalently, $x j \in N_{G}\left(E^{\times}\right)$. We have $N_{G}\left(E^{\times}\right) \cap \mathbf{J}=E^{\times}\left(N_{G}\left(E^{\times}\right) \cap J^{1}\right)$. As $J^{1}$ is a pro- $p$ group and $\operatorname{Aut}(E \mid F)=$ $N_{G}\left(E^{\times}\right) / E^{\times}$has order prime to $p$, the intersection $N_{G}\left(E^{\times}\right) \cap J^{1}$ is contained in $E^{\times}$, whence the sum (4.1.2) is effectively taken over $\operatorname{Aut}(E \mid F)$. The result now follows from the lemma.

4.2. For the next step, we are given a cyclic sub-extension $K / F$ of $E / F$, of degree $d$, with $\Sigma=\operatorname{Gal}(K / F)$. The pair $(E / K, \xi)$ is then admissible and we may form the representation $\rho={ }_{K} \pi_{\xi} \in \mathcal{A}_{m}^{\text {et }}(K)$, where $m=n / d$.

The representation $\rho$ contains an extended maximal simple type $\left(\mathbf{J}_{K}, \Lambda_{K}\right)$, say, based on a simple stratum in $\mathrm{M}_{m}(K)$. We use this to define a Glauberman sign $\epsilon_{K}= \pm 1$, as in (4.1.1). This gives us:

Lemma 4.2. The representation $\Lambda_{K}$ satisfies

$$
\operatorname{tr} \Lambda_{K}(h)=\epsilon_{K} \xi(h),
$$

for all $h \in E^{\times}$with $v_{E}(h)$ relatively prime to $n$.

4.3. We now change notation, and let $\pi=\mathrm{A}_{K / F} \rho$ be the representation of $G$ which is automorphically induced by $\rho$. By Proposition $3.4, \pi \in \mathcal{A}_{n}^{\text {et }}(F)$ and $\pi={ }_{F} \pi_{\mu \xi}$, for some tamely ramified character $\mu=_{K / F} \mu_{\xi}$ of $E^{\times}$. In particular, $\pi$ contains an extended maximal simple $(\mathbf{J}, \Lambda)$, based on the same simple stratum $[\mathfrak{A}, l, 0, \beta]$ as in section 4.1. Replacing $\xi$ by $\mu \xi$ does not change the constant $\epsilon_{F}= \pm 1$ of (4.1.1), since $\epsilon_{F}$ depends only on the action of $E^{\times}$on $Q$, which in turn depends only on $\xi \mid U_{E}^{1}$.

We recall the automorphic induction equation. The cyclic extension $K / F$ corresponds, via class field theory, to a cyclic group $Y_{K / F}$ of characters of $F^{\times}$. We choose a generator $\varkappa$ of $Y_{K / F}$; following the conventions of [3], we form the $\varkappa$ twisted character $\Xi_{\pi}^{\varkappa}$ of $\pi$, relative to the $\varkappa$-operator which acts as the identity on the $\theta$-isotypic subspace of $\pi$. Writing $G_{K}$ for the $G$-centralizer of $K^{\times}$, we have [16] 3.11], [17,

$$
\Xi_{\pi}^{\varkappa}(h)=c \boldsymbol{\delta}(h) \sum_{\sigma \in \Sigma} \operatorname{tr} \rho^{\sigma}(h), \quad h \in G_{K} \cap G_{\mathrm{reg}}^{\mathrm{ell}},
$$

where $G_{\mathrm{reg}}^{\mathrm{ell}}$ is the set of elliptic regular elements of $G$. In this formula, $\delta$ is a transfer factor: in the conventions of [16], $\boldsymbol{\delta}(h)=\Delta^{2}(h) / \Delta^{1}(h)$. The factor $c$ is some non-zero constant.

We prove:

Theorem 4.3. Let $(E / F, \xi) \in P_{n}(F)$, and suppose that $E / F$ is totally ramified. Let $K / F$ be a cyclic sub-extension of $E / F$. The character $\mu={ }_{K / F} \mu_{\xi}$ satisfies

$$
\mu(h)=\epsilon_{F} \epsilon_{K} c \boldsymbol{\delta}(h),
$$

for every $h \in E^{\times}$such that $v_{E}(h)$ is relatively prime to $n$.

Proof. Let $\Lambda$ be the unique irreducible representation of $\mathbf{J}=E^{\times} J^{1}(\beta, \mathfrak{A})$ which occurs in $\pi$ and contains the simple character $\theta$. With our definition of $\Xi_{\pi}^{\varkappa}$, we 
have [3, 15.8]:

$$
\Xi_{\pi}^{\varkappa}(h)=\sum_{x \in G / \mathbf{J}} \varkappa\left(\operatorname{det} x^{-1}\right) \operatorname{tr} \Lambda\left(x^{-1} h x\right) .
$$

We evaluate this sum exactly as in Proposition 4.1 to get

$$
\Xi_{\pi}^{\varkappa}(h)=\epsilon_{F} \sum_{\alpha \in \operatorname{Aut}(E \mid F)} \varkappa(\operatorname{det} \alpha) \mu^{\alpha} \xi^{\alpha}(h),
$$

valid for $h \in E^{\times}$of valuation prime to $n$.

We consider the character values $\operatorname{tr} \rho^{\sigma}(h)$, for $\sigma \in \Sigma$ and $h$ as before.

Lemma 4.3. Let $h \in E^{\times}$have valuation prime to $n$, and let $\sigma \in \Sigma$. Then $\operatorname{tr} \rho^{\sigma}(h)=0$ unless $\sigma$ extends to an F-automorphism of $E$.

Proof. For each $\sigma \in \Sigma$, we choose $t_{\sigma} \in G$ such that conjugation by $t_{\sigma}$ induces the action of $\sigma$ on $K$. Suppose that $\operatorname{tr} \rho^{\sigma}(h)=\operatorname{tr} \rho\left(t_{\sigma} h t_{\sigma}^{-1}\right) \neq 0$. As in Proposition 4.1, there exists $x \in G_{K}$ such that $x t_{\sigma} h t_{\sigma}^{-1} x^{-1} \in E^{\times}$, which implies $x t_{\sigma} \in N_{G}\left(E^{\times}\right)$and hence the result.

We can apply the considerations of section 4.2 to the representation $\rho^{\sigma}$ in place of $\rho$. In particular, $\rho^{\sigma}$ gives rise to a constant $\epsilon_{K}^{(\sigma)}= \pm 1$. For $h$ as before and $\sigma \in \Sigma$, we obtain

$$
\operatorname{tr} \rho^{\sigma}(h)=\sum_{\substack{\alpha \in \operatorname{Aut}(E \mid F), \alpha \mid K=\sigma}} \epsilon_{K}^{(\sigma)} \xi^{\alpha}(h),
$$

with the understanding that the sum is zero when the index set is empty. In all, therefore,

$$
\epsilon_{F} \sum_{\alpha \in \operatorname{Aut}(E \mid F)} \varkappa(\operatorname{det} \alpha) \mu^{\alpha} \xi^{\alpha}(h)=c \boldsymbol{\delta}(h) \sum_{\alpha \in \operatorname{Aut}(E \mid F)} \epsilon_{K}^{(\alpha \mid K)} \xi^{\alpha}(h) .
$$

This relation continues to hold on replacing $h$ by $h u$, for $u \in U_{E}^{1}$. The definition [16] shows readily that $\boldsymbol{\delta}(h u)=\boldsymbol{\delta}(h)$, so

$$
\epsilon_{F} \sum_{\alpha \in \operatorname{Aut}(E \mid F)} \varkappa(\operatorname{det} \alpha) \mu^{\alpha} \xi^{\alpha}(h) \xi^{\alpha}(u)=c \boldsymbol{\delta}(h) \sum_{\alpha \in \operatorname{Aut}(E \mid F)} \epsilon_{K}^{(\alpha \mid K)} \xi^{\alpha}(h) \xi^{\alpha}(u),
$$

for $h \in E^{\times}$with $v_{E}(h)$ prime to $n$ and $u \in U_{E}^{1}$. Since $(E / F, \xi)$ is admissible and $E / F$ is totally ramified, the characters $\xi^{\alpha}$ of $U_{E}^{1}$ are distinct, as $\alpha$ ranges over $\operatorname{Aut}(E \mid F)$. We therefore multiply the last equation by $\xi(u)^{-1}$ and integrate over $U_{E}^{1}$ to get the result.

Remark. The formula (4.3.1) determines the character $\mu$ completely. However, we cannot give it an explicit form since the value of the constant $c$ is unknown. Note that the function $\boldsymbol{\delta}(h)$ depends only on $n$ and the extension $K / F$ (modulo certain normalizations independent of $\xi$ ). We will show elsewhere that, with our definition of the twisted trace, the constant $c$ depends only on the restriction $\xi \mid U_{E}^{1}$, whence the same is true of $\mu$. We do not use that property in this paper. 
4.4. We now specialize to the case where $n$ is odd, and prove Theorem B of the Introduction:

Theorem 4.4. Let $(E / F, \xi) \in P_{n}(F)$. Suppose that $n$ is odd and that $E / F$ is totally ramified. We then have

$$
{ }_{F} \mu_{\xi}=\delta_{E / F} \circ \mathrm{N}_{E / F} .
$$

The discriminant character $\delta_{E / F}$ is easy to describe: it is given by a Jacobi symbol

$$
\delta_{E / F}(x)=\left(\frac{q}{n}\right)^{v_{F}(x)}, \quad x \in F^{\times}
$$

(see, for example, [2, 10.1.6]).

4.5. The first step in the proof of Theorem 4.4 is:

Proposition 4.5. Let $(E / F, \xi) \in P_{n}(F)$, with $n$ odd and $E / F$ totally ramified. Let $K / F$ be a cyclic sub-extension of $E / F$. The character $K_{K / F} \mu_{\xi}$ is then trivial.

Proof. Since $E / F$ is totally tamely ramified, there exists a prime element $\varpi$ of $E$ such that $\varpi^{n}=\varpi_{F}$ is a prime element of $F$. We consider the transfer factor $\delta\left(\varpi^{r}\right)$, for integers $r$ relatively prime to $n$.

Lemma 4.5. As $r$ ranges over the integers relatively prime to $n$, the function $r \mapsto \boldsymbol{\delta}\left(\varpi^{r}\right)$ is constant.

Proof. To compute the transfer factors, we follow the recipes in [16]. We need to fix a generator $\sigma_{0}$ of $\Sigma$ and an element $e_{m}$ which, since $n$ is odd, we may set equal to 1 . The character relation in Theorem 4.3 shows that the $|\boldsymbol{\delta}(h)|$ is constant on $G$-special elements of $E^{\times}$. In the definition of the transfer factor, $|\delta(h)|=\Delta^{1}(h)^{-1}$, so it is enough to show that the function $r \mapsto \Delta^{2}\left(\varpi^{r}\right)$ is constant.

We have $\sigma_{0}\left(\varpi^{m}\right)=\eta \varpi^{m}$, for some $d$-th root of unity $\eta$. For $0 \leqslant i \leqslant d-1$, the eigenvalues of $\sigma_{0}^{i}(\varpi)$ are therefore $\zeta^{i} \varpi$, where $\zeta$ ranges over the $m$-th roots of $\eta$. In the notation of [16], we have $\Delta^{2}=\varkappa \circ \widetilde{\Delta}$ and

$$
\widetilde{\Delta}(\varpi)=\varpi^{m^{2} d(d-1) / 2} \prod_{0 \leqslant i<j \leqslant d-1} \prod_{\zeta_{1}, \zeta_{2}}\left(\zeta_{1}^{i}-\zeta_{2}^{j}\right),
$$

where $\zeta_{1}, \zeta_{2}$ range independently over the $m$-th roots of $\eta$.

We can describe the double product

$$
\Pi=\prod_{0 \leqslant i<j \leqslant d-1} \prod_{\zeta_{1}, \zeta_{2}}\left(\zeta_{1}^{i}-\zeta_{2}^{j}\right)
$$

in another way. Let $\eta_{1}, \eta_{2}$ range independently over the $n$-th roots of unity. Then $\Pi$ is the product of all differences $\left(\eta_{1}-\eta_{2}\right)$, subject to two conditions: $\eta_{1}^{m} \neq \eta_{2}^{m}$ and exactly one of the possibilities $\pm\left(\eta_{1}-\eta_{2}\right)$ occurs.

We apply the same analysis to $\widetilde{\Delta}\left(\varpi^{r}\right),(r, n)=1$, to obtain

$$
\widetilde{\Delta}\left(\varpi^{r}\right)= \pm \varpi^{r m^{2} d(-1) / 2} \Pi .
$$

Since $n$ is odd and $K / F$ is totally ramified, we have $\varkappa\left(\varpi_{F}\right)=1$. Thus

$$
\varkappa\left(\varpi^{m^{2} d(d-1) / 2}\right)=\varkappa\left(\varpi_{F}^{m(d-1) / 2}\right)=1 .
$$

Likewise, $\varkappa(-1)=1$, so $\Delta^{2}\left(\varpi^{r}\right)=\varkappa(\Pi)=\Delta^{2}(\varpi)$, as required. 
Returning to the proof of the proposition, we have $\mu\left(\varpi^{r}\right)=c \epsilon_{F} \epsilon_{K} \delta\left(\varpi^{r}\right)$, whenever $(r, n)=1$. The character $\mu$ is trivial on $U_{E}^{1}$ and also on $F^{\times}$, since $\delta_{K / F}=1$. In effect, therefore, $\mu$ is a character of the group $E^{\times} / F^{\times} U_{E}^{1} \cong \mathbb{Z} / n \mathbb{Z}$. The lemma shows that it is constant on the generators of this group and, since $n$ is odd, $\mu$ must be trivial.

4.6. The proof of Theorem 4.4 relies on an auxiliary result (which does not require our assumption that $n$ is odd).

Theorem 4.6. Let $(E / F, \xi) \in P_{n}(F)$, and suppose that $E / F$ is totally ramified. Let $L / F$ be unramified of degree $d$, with $(d, n)=1$. Write $\xi_{L}=\xi \circ \mathrm{N}_{E L / E}$. The pair $\left(E L / L, \xi_{L}\right)$ is then admissible and

$$
{ }_{L} \pi_{\xi_{L}}=\mathrm{b}_{L / F}\left({ }_{F} \pi_{\xi}\right) .
$$

We shall prove this in the following paragraphs. We first use it to complete the proof of Theorem 4.4.

We proceed by induction on $n$. Let $\ell$ be the least prime divisor of $n$ and let $K / F$ be the unique sub-extension of $E / F$ of degree $\ell$. By the induction hypothesis, we have

$$
\delta_{E / K} \cdot{ }_{K} \pi_{\xi}=\mathcal{L}\left(\operatorname{Ind}_{E / K} \xi\right)
$$

The character $\delta_{E / K}$ is unramified and hence of the form $\delta_{E / K}=\phi \circ \mathrm{N}_{K / F}$, for some unramified character $\phi$ of $F^{\times}$, having the same order as $\delta_{E / K}$. In particular, $\phi^{2}=1$.

If $K / F$ is cyclic, we have $\delta_{K / F}=1$ and, by Proposition $4.5, \mathrm{~A}_{K / F}\left({ }_{K} \pi_{\xi}\right)={ }_{F} \pi_{\xi}$ whence

$$
\phi \cdot{ }_{F} \pi_{\xi}=\mathrm{A}_{K / F}\left(\delta_{E / K} \cdot{ }_{K} \pi_{\xi}\right)=\mathcal{L}\left(\operatorname{Ind}_{E / F} \xi\right) .
$$

However, $\delta_{E / F}=\delta_{K / F}^{n / \ell} \delta_{E / K} \mid F^{\times}=\phi^{\ell}=\phi$, as required for Theorem 4.4.

We therefore assume that $K / F$ is not cyclic. We put $\sigma=\operatorname{Ind}_{E / F} \xi$. We let $L / F$ be the unramified extension obtained by adjoining to $F$ a primitive $\ell$-th root of unity. Let $k=[L: F]$; since $k<\ell$ and $\ell$ is the least prime divisor of $n$, we have $(k, n)=1$. Applying Theorem 4.6, we get

$$
{ }_{L} \pi_{\xi_{L}}=\mathrm{b}_{L / F}\left({ }_{F} \pi_{\xi}\right) .
$$

The extension $L K / L$ is cyclic so, by the first case,

$$
{ }_{L} \pi_{\xi_{L}}=\delta_{E L / L} \cdot \mathcal{L}\left(\sigma_{L}\right),
$$

where $\sigma_{L}=\sigma \mid \mathcal{W}_{L}=\operatorname{Ind}_{E L / L} \xi_{L}$. The character $\delta_{E L / L}$ is just $\delta_{E / F} \circ \mathrm{N}_{L / F}$, so

$$
{ }_{L} \pi_{\xi_{L}}=\mathrm{b}_{L / F}\left(\delta_{E / F} \cdot \mathcal{L}(\sigma)\right) .
$$

Comparing (4.6.1), (4.6.2), we get ${ }_{F} \pi_{\xi}=\chi \delta_{E / F} \cdot \mathcal{L}(\sigma)$, for some unramified character $\chi$ of $F^{\times}$such that $\chi^{k}=1$ [8] 2.6]. However, comparing central quasicharacters, we get

$$
\xi=\chi^{n} \delta_{E / F}^{n} \operatorname{det} \sigma=\chi^{n} \delta_{E / F}^{n+1} \xi
$$

since $n$ is odd, this reduces to $\chi^{n}=1$. We also have $\chi^{k}=1$ and $(k, n)=1$, so $\chi=1$, as required.

This completes the proof of Theorem 4.4. 
4.7. We now prove Theorem 4.6. We abbreviate $\pi={ }_{F} \pi_{\xi}, \pi_{L}={ }_{L} \pi_{\xi_{L}}$ and $\Pi=$ $\mathrm{b}_{L / F} \pi$. In this notation, we have to show that $\pi_{L}=\Pi$.

The endo-class $\Theta\left(\pi_{L}\right)$ is given by $\Theta\left(\pi_{L}\right)=\mathrm{R}_{E L / L}\left(\xi_{L} \mid U_{E L}^{1}\right)$ whence

$$
\mathrm{R}_{L / F} \Theta\left(\pi_{L}\right)=\Theta(\pi)
$$

thus $\Theta\left(\pi_{L}\right)$ is an $L / F$-lift of $\Theta(\pi)$. (Indeed, it is the unique $L / F$-lift of $\Theta(\pi)$.) On the other hand, this equals $\Theta(\Pi)$, by [6, (7.1)]. The representations $\Pi, \pi_{L}$ have the same central quasicharacter, namely $\omega_{\pi} \circ \mathrm{N}_{L / F}$. Since $E / F$ is totally ramified, we deduce:

Lemma 4.7. There is an unramified character $\chi$ of $L^{\times}$such that $\chi^{n}=1$ and $\pi_{L}=\chi \Pi$.

We have to show that $\chi=1$.

4.8. We pass to the group $G(L)=\mathrm{GL}_{n}(L)$, and set $\Sigma=\operatorname{Gal}(L / F)$. The pair $\left(L E / L, \xi_{L}\right)$ gives an extended maximal simple type $\left(\mathbf{J}_{L}, \Lambda_{L}\right)$ in $\pi_{L}$, which we now describe. Write $\mathfrak{A}_{L}=\mathfrak{A} \otimes_{\mathfrak{o}_{F}} \mathfrak{o}_{L}$; the quadruple $\left[\mathfrak{A}_{L}, l, 0, \beta\right]$ is then a simple stratum in $A(L)=\mathrm{M}_{n}(L)$ and, setting $\psi_{L}=\psi_{F} \circ \operatorname{Tr}_{L / F}$, there is a simple character $\theta_{L} \in \mathcal{C}\left(\mathfrak{A}_{L}, \beta, \psi_{L}\right)$ such that $\mathcal{E}_{L}\left(\theta_{L}\right)$ is the unique $L / F$-lift of $\mathcal{E}_{F}(\theta)$ [3. (11.2) et seq]. We may therefore take $\mathbf{J}_{L}=E L^{\times} J^{1}\left(\beta, \mathfrak{A}_{L}\right)$ and assume that $\Lambda_{L} \mid J^{1}\left(\beta, \mathfrak{A}_{L}\right)=\eta_{L}$, the unique irreducible representation of $J^{1}\left(\beta, \mathfrak{A}_{L}\right)$ containing $\theta_{L}$.

The character $\theta_{L}$ is $\Sigma$-stable (remark following [3, (11.3)]), and the same therefore applies to $\eta_{L}$. It follows (cf. Lemma 4.1) that $\Lambda_{L}$ is $\Sigma$-stable.

We choose a generator $\sigma$ of $\Sigma$. The quasicharacter $\xi_{L}$ of $L E^{\times}$is $\Sigma$-stable, so we can extend it to a quasicharacter $\tilde{\xi}_{L}$ of $L E^{\times} \rtimes \Sigma$ by setting $\tilde{\xi}_{L} \mid \Sigma=1$. Likewise, $\Lambda_{L}$ admits an extension to a representation of $\mathbf{J}_{L} \rtimes \Sigma$.

Lemma 4.8. Let $\widetilde{\Lambda}_{L}$ be a representation of $\mathbf{J}_{L} \rtimes \Sigma$ which extends $\Lambda_{L}$. There is a non-zero constant $\epsilon_{L}^{\prime}$ such that

$$
\operatorname{tr} \widetilde{\Lambda}_{L}\left(\varpi^{r} \zeta x \sigma\right)=\epsilon_{L}^{\prime} \tilde{\xi}_{L}\left(\varpi^{r} \zeta x \sigma\right)=\epsilon_{L}^{\prime} \xi_{L}\left(\varpi^{r} \zeta x\right),
$$

for all integers $r$ such that $(r, n)=1$ and all $\zeta \in \boldsymbol{\mu}_{F}, x \in U_{E}^{1}$.

Proof. Set $J_{L}^{1}=J^{1}\left(\beta, \mathfrak{A}_{L}\right)$ and $H_{L}^{1}=H^{1}\left(\beta, \mathfrak{A}_{L}\right)$. Observe that the assertion to be proved only concerns the restriction of $\widetilde{\Lambda}_{L}$ to $E^{\times} J_{L}^{1}$.

Write $\tilde{\theta}_{L}=\tilde{\xi}_{L} \mid H_{L}^{1} \rtimes \Sigma$. There is a unique irreducible representation $\tilde{\eta}_{L}$ of $J_{L}^{1} \rtimes \Sigma$ which restricts, on $H_{L}^{1} \rtimes \Sigma$, to a multiple of $\tilde{\theta}_{L}$. This representation $\tilde{\eta}_{L}$ is inflated from a representation of the finite group $J_{L}^{1} \rtimes \Sigma / \operatorname{Ker} \theta_{L}$ of order $d p^{s}$, for some integer $s$. The group $E^{\times}$acts, by conjugation, as a cyclic group of automorphisms of order $n$, which is prime to $d p^{s}$. This action fixes $\tilde{\eta}_{L}$ and so gives rise to a Glauberman sign $\epsilon_{L}= \pm 1$. Thus there exists a unique representation $\bar{\Lambda}_{L}$ of $E^{\times} J_{L}^{1} \rtimes \Sigma$ such that

$$
\operatorname{tr} \bar{\Lambda}_{L}\left(\varpi^{r} \zeta x \sigma\right)=\epsilon_{L} \tilde{\xi}_{L}\left(\varpi^{r} \zeta x \sigma\right)=\epsilon_{L} \xi_{L}\left(\varpi^{r} \zeta x\right),
$$

for $(r, n)=1, \zeta \in \boldsymbol{\mu}_{F}, x \in U_{E}^{1}$.

Finally, we note that $\widetilde{\Lambda}_{L} \mid E^{\times} J_{L}^{1} \rtimes \Sigma$ is of the form $\phi \otimes \bar{\Lambda}_{L}$, for some character $\phi$ of $\Sigma$. The result follows, with $\epsilon_{L}^{\prime}=\epsilon_{L} \phi(\sigma)$.

We put

$$
\tilde{\pi}_{L}=c-\operatorname{Ind}_{\mathbf{J}_{L} \rtimes \Sigma}^{G(L) \rtimes \Sigma} \widetilde{\Lambda}_{L} .
$$


This is an irreducible representation of $G(L) \rtimes \Sigma$ extending $\pi_{L}$.

Again we say that an element $h$ of $\mathbf{J}_{L}$ is $G(L)$-special if $v_{L}(\operatorname{det} h)$ is relatively prime to $n$.

Proposition 4.8. Let $h \in \mathbf{J}_{L}$ be $G(L)$-special. Then

$$
\operatorname{tr} \tilde{\pi}_{L}(h \sigma)=\epsilon_{F} \epsilon_{L}^{\prime} \operatorname{tr} \pi\left(\mathcal{N}_{\sigma} h\right),
$$

where $\mathcal{N}_{\sigma} h$ is the $\sigma$-norm of $h$ in $G$.

Proof. Let $h \in \mathbf{J}_{L}$ be $G(L)$-special. We have $v_{F}\left(\operatorname{det} \mathcal{N}_{\sigma} h\right)=d v_{L}(\operatorname{det} h)$; since $(d, n)=1$, the element $\mathcal{N}_{\sigma} h$ is $G$-special.

We write $J_{L}^{1}=J^{1}\left(\beta, \mathfrak{A}_{L}\right)$. We have $h=j h_{1}$, where $h_{1} \in L E^{\times}$and $j \in J_{L}^{1}$. The element $h \sigma$ acts on $J_{L}^{1}$, by conjugation, as an automorphism of finite order relatively prime to $p$. It follows that $h \sigma$ is $J_{L}^{1}$-conjugate to an element $j_{1} h_{1} \sigma$, where $j_{1} \in J_{L}^{1}$ commutes with $h_{1} \sigma$. That is, $h \sigma$ is $J_{L}^{1}$-conjugate to an element of $L E^{\times} \sigma$. If $h \in L E^{\times}$, we have $\mathcal{N}_{\sigma} h=\mathrm{N}_{L E / E}(h)$ so $\mathcal{N}_{\sigma}(h)$ is an elliptic regular, $G$-special element of $G$.

Let $h, h^{\prime} \in L E^{\times}$be $G(L)$-special, and suppose that $h^{\prime} \sigma=x^{-1} h \sigma x$, for some $x \in G(L)$. The $\sigma$-norms of $h, h^{\prime}$ are then conjugate by the same element $x$, whence $\mathrm{N}_{L E / E}\left(h^{\prime}\right)=x^{-1} \mathrm{~N}_{L E / E}(h) x$. Each of these norms generates $E / F$. We conclude that conjugation by $x$ is an $L$-automorphism of $L E$, which we now denote by $\alpha$. This satisfies $\alpha(\varpi)=\zeta \varpi$, for some $n$-th root of unity $\zeta$ in $L$.

We consider the element $x^{-1} h \sigma x=\alpha(h) x^{-1} \sigma x$. Conjugation by the element $x^{-1} \sigma x \sigma^{-1}$ is an $L$-automorphism of $L E$ mapping $\varpi$ to $\sigma^{-1}\left(\zeta^{-1}\right) \zeta \varpi$. By definition, we have $\alpha(h) x^{-1} \sigma x \sigma^{-1}=h^{\prime}$, so $x^{-1} \sigma x \sigma^{-1} \in \mathbf{J}_{L}$. The automorphism of $L E$ induced by $x^{-1} \sigma x \sigma^{-1}$ is therefore trivial, so $\sigma$ commutes with $\alpha$ and therefore $\sigma(\zeta)=\zeta$. It follows that $\alpha \in \operatorname{Aut}(E L / L) \cong \operatorname{Aut}(E \mid F)$. Therefore, if $h \in L E^{\times}$is $G(L)$-special, we have

$$
\begin{aligned}
\operatorname{tr} \widetilde{\pi}(h \sigma) & =\epsilon_{L}^{\prime} \sum_{\alpha \in \operatorname{Aut}(E \mid F)} \xi_{L}(\alpha(h)) \\
& =\epsilon_{L}^{\prime} \sum_{\alpha \in \operatorname{Aut}(E \mid F)} \xi\left(\alpha\left(\mathrm{N}_{L E / E}(h)\right)\right) \\
& =\epsilon_{F}^{\prime} \epsilon_{L} \operatorname{tr} \pi\left(\mathcal{N}_{\sigma} h\right),
\end{aligned}
$$

by Proposition 4.1, and the result follows.

4.9. We return to the representation $\Pi=\mathrm{b}_{L / F} \pi=\chi \pi_{L}$, where $\chi$ is unramified and $\chi^{n}=1$. We extend $\Pi$ to a representation $\widetilde{\Pi}$ of $G(L) \rtimes \Sigma$; there is then a non-zero constant $c$ such that

$$
\operatorname{tr} \widetilde{\Pi}(h \sigma)=c \operatorname{tr} \pi\left(\mathcal{N}_{\sigma} h\right),
$$

for all $h \in G(L)$ such that $\mathcal{N}_{\sigma} h$ is a quasi-regular element of $G$ [3, Appendix]. In particular, if $h \in L E^{\times}$is $G(L)$-special, Proposition 4.8 gives

$$
\epsilon_{F} \epsilon_{L}^{\prime} \operatorname{tr} \widetilde{\pi}_{L}(h \sigma)=\operatorname{tr} \pi\left(\mathcal{N}_{\sigma} h\right)=c^{-1} \operatorname{tr} \widetilde{\Pi}(h \sigma)=c^{-1} \chi(\operatorname{det} h) \operatorname{tr} \widetilde{\pi}_{L}(h \sigma) .
$$

In other words, there is a constant $c^{\prime}$ such that

$$
\chi(\operatorname{det} h) \operatorname{tr} \widetilde{\pi}_{L}(h \sigma)=c^{\prime} \operatorname{tr} \widetilde{\pi}_{L}(h \sigma)
$$


for all $G(L)$-special elements $h$ of $L E^{\times}$. We now write $\chi=\phi \circ \mathrm{N}_{L / F}$, for some unramified character $\phi$ of $F^{\times}$satisfying $\phi^{n}=1$ (as we may, since $(d, n)=1$ ). Invoking Proposition 4.8 again, there is a constant $c^{\prime \prime}$ such that

$$
\phi(\operatorname{det} x) \operatorname{tr} \pi(x)=c^{\prime \prime} \operatorname{tr} \pi(x),
$$

for all $G$-special $x \in E^{\times}$with $v_{F}(\operatorname{det} x) \equiv 0(\bmod d)$.

The representation $\pi$ is totally ramified so, given $a \in \mathbb{Z}$, there exists $g \in G$ with $v_{F}(\operatorname{det} g)=a$ and $\operatorname{tr} \pi(g) \neq 0$. By Proposition 4.1, if $(a, n)=1$, we can take $g \in E^{\times}$. Therefore $\phi$ must be constant on $\left(F^{\times}\right)^{d} U_{F}$, whence $\phi^{d}=1$. Since $\phi^{n}=1$ and $(n, d)=1$, we have $\phi=1$, as required.

This completes the proof of Theorem 4.6.

\section{Appendix. Characters of tame extensions}

We gather here some elementary results concerning quasicharacters of finite, tamely ramified extensions of the base field $F$. While these are, broadly speaking, well known, they are also widely forgotten. They are scattered in literature which is now rather old, often in the wrong degree of generality. It seems more satisfactory, therefore, to collect them in this brief appendix.

For our present purposes, we define a representation $\sigma \in \mathcal{G}_{n}^{0}(F)$ to be essentially tame if its restriction to the wild inertia subgroup of $\mathcal{W}_{F}$ is a sum of characters.

A.1. Let $E / F$ be a finite, Galois, tamely ramified field extension and put $\Gamma=$ $\operatorname{Gal}(E / F)$. The basic fact, on which all depends, is that the $\Gamma$-modules $U_{E}^{n}, n \geqslant 1$, are cohomologically trivial. The same applies to $U_{E}$ when $E / F$ is unramified. (See 26] for a comprehensive account of such matters.) Consequently:

Lemma. (1) Let $E / F$ be a finite, Galois, tamely ramified field extension, and let $K / F$ be a sub-extension with $\Delta=\operatorname{Gal}(E / K)$. Let $\chi$ be a character of $U_{E}^{n+1}$ with $n \geq 0$ (or $n \geqslant-1$ if $E / K$ is unramified). Then $\chi$ factors through $\mathrm{N}_{E / K}$ if and only if $\chi^{\delta}=\chi$, for all $\delta \in \Delta$.

(2) Suppose, in (1), that $\chi \mid U_{E}^{n+1}$ factors through $\mathrm{N}_{E / K}$. Let $e=e(E \mid K)$ and $m=[n / e]$, so that $U_{E}^{n+1} \cap K=U_{K}^{m+1}=\mathrm{N}_{E / K}\left(U_{E}^{n+1}\right)$. There is then a unique character $\eta$ of $U_{K}^{m+1}$ such that $\chi \mid U_{E}^{n+1}=\eta \circ \mathrm{N}_{E / K}$.

(3) Let $E / F$ be a finite, tamely ramified field extension and let $\chi$ be a character of $U_{E}^{n}$, for some $n \geq 0$. There exists a unique sub-extension $E_{0} / F$ of $E / F$ such that $\chi$ factors through $\mathrm{N}_{E / E_{0}}$ and which is minimal for this property.

In (3), the field $E_{0}$ arises as follows. Let $L / F$ be the normal closure of $E / F$ so that, in particular, $L / E$ is unramified. Set $\chi_{L}=\chi \circ \mathrm{N}_{L / E}$, and let $\Delta$ be the subgroup of $\operatorname{Gal}(L / F)$ which fixes $\chi_{L} \mid U_{L}^{n}$. Then $E_{0}=L^{\Delta}$.

A.2. We return to the notion of "admissible pair", defined in the Introduction. The first property of these pairs is:

Proposition. Let $(E / F, \xi)$ be an admissible pair. Then $\operatorname{Ind}_{E / F} \xi$ is irreducible and essentially tame.

Proof. Let $L / F$ be the normal closure of $E / F$. In particular, $L / E$ is unramified. We put $\Gamma=\operatorname{Gal}(L / F), \Delta=\operatorname{Gal}(L / E)$.

Suppose, for a contradiction, that $\operatorname{Ind}_{E / F} \xi$ is reducible. By Mackey's criterion, therefore, there exists $g \in \Gamma, g \notin \Delta$, such that $\xi$ and $\xi^{g}$ have the same restriction 
to $\mathcal{W}_{L}$. This says that the quasicharacter $\xi \circ \mathrm{N}_{L / E}$ of $L^{\times}$is invariant under the subgroup $\Omega=\langle\Delta, g\rangle$ of $\Gamma$ generated by $\Delta$ and this element $g$. The restriction of $\xi$ to $U_{E}^{1}$ therefore factors through $\mathrm{N}_{E / K}$, where $K=L^{\Omega}$. Since $(E / F, \xi)$ is admissible, the extension $E / K$ is unramified. Therefore $L / K$ is unramified. The group $\Omega$ therefore stabilizes $E$ and, by definition, it stabilizes the quasicharacter $\xi \circ \mathrm{N}_{L / E}$ of $L^{\times}$. This quasicharacter therefore factors through $\mathrm{N}_{L / K}$ and so $\xi$ factors through $\mathrm{N}_{E / K}$. Since $(E / F, \xi)$ is admissible, we have $K=E$ and the desired contradiction. The final assertion is immediate.

A.3. Let $(E / F, \xi)$ be an admissible pair; the equivalence class of the representation $\sigma=\operatorname{Ind}_{E / F} \xi$ depends only on the $F$-isomorphism class of $(E / F, \xi)$ and $\sigma$ is surely essentially tame. Thus we have a canonical map

$$
\begin{gathered}
P_{n}(F) \longrightarrow \mathcal{G}_{n}^{\text {et }}(F), \\
(K / F, \xi) \longmapsto \operatorname{Ind}_{K / F} \xi,
\end{gathered}
$$

for every $n \geq 1$.

Theorem. For every $n \geq 1$, the map (A.3.1) is a bijection.

Proof. Let $\sigma \in \mathcal{G}_{n}^{\text {et }}(F)$ act on the vector space $V$. Let $L / F$ be a finite, tamely ramified, Galois extension such that $\sigma \mid \mathcal{W}_{L}$ is a direct sum of quasicharacters. Let $\eta$ be a component of $\sigma \mid \mathcal{W}_{L}$, viewed as a quasicharacter of $L^{\times}$, and let $\Delta$ be the subgroup of $\operatorname{Gal}(L / F)$ which fixes $\eta \mid U_{L}^{1}$. Set $K=L^{\Delta}$. The implied representation $\tau$ of $\mathcal{W}_{K}$ on the isotypic space $V^{\eta}$ is irreducible and, by Clifford theory, $\sigma=\operatorname{Ind}_{K / F} \tau$. On the other hand, there is a quasicharacter $\phi$ of $K^{\times}$such that $\phi \circ \mathrm{N}_{L / K}$ agrees with $\eta$ on $U_{K}^{1}$, so $\tau \cong \phi \otimes \rho$, for some irreducible representation $\rho$ of $\mathcal{W}_{K}$ trivial on $\mathcal{W}_{L}$. Thus $\rho$ can be viewed as an irreducible representation of $\Delta \cong \mathcal{W}_{K} / \mathcal{W}_{L}$. The Galois extension $L / K$ is tamely ramified, so $\rho$ is of the form $\rho=\operatorname{Ind}_{E / K} \mu$, where $E / K$ is an unramified extension in $L$ and $\mu$ is a tamely ramified quasicharacter of $E^{\times}$. We put $\xi=\mu \cdot\left(\phi \circ \mathrm{N}_{E / K}\right)$ to get $\sigma=\operatorname{Ind}_{E / F} \xi$.

We next show that the pair $(E / F, \xi)$ is admissible. Clearly, condition (1) in the definition is satisfied, since $\operatorname{Ind}_{E / F} \xi=\sigma$ is irreducible. Let $K^{\prime}$ be an extension of $F$ in $E$ and $\phi^{\prime}$ a character of $U_{K^{\prime}}^{1}$ such that $\phi^{\prime} \circ \mathrm{N}_{E / K^{\prime}}=\xi \mid U_{E}^{1}$. The group $\operatorname{Gal}\left(L / K^{\prime}\right)$ therefore stabilizes $\xi \circ \mathrm{N}_{L / E}\left|U_{L}^{1}=\eta\right| U_{L}^{1}$, so $K^{\prime} \supset K$ whence $E / K$ is unramified. Thus $(E / F, \xi)$ is admissible and the map (A.3.1) is surjective.

Finally, we have to show that (A.3.1) is injective. With $\sigma$ and $(E / F, \xi)$ as before, suppose we have another admissible pair $\left(E^{\prime} / F, \xi^{\prime}\right)$ such that $\operatorname{Ind}_{E^{\prime} / F} \xi^{\prime}=\sigma$. Enlarging $L$ if need be, we can assume that both $E$ and $E^{\prime}$ are contained in $L$. The quasicharacters $\xi \circ \mathrm{N}_{L / E}, \xi^{\prime} \circ \mathrm{N}_{L / E^{\prime}}$ of $L^{\times}$are then conjugate under $\operatorname{Gal}(L / F)$. Replacing $\left(E^{\prime} / F, \xi^{\prime}\right)$ by an $F$-isomorphic pair, we can assume that $\xi^{\prime} \circ \mathrm{N}_{L / E^{\prime}}=$ $\xi \circ \mathrm{N}_{L / E}$. Let $\Delta$ be the subgroup of $\operatorname{Gal}(L / F)$ fixing these quasicharacters on $U_{L}^{1}$ and set $K=L^{\Delta}$ : this is indeed the same as the field $K$ attached to $\sigma=\operatorname{Ind}_{E / F} \xi$ in the first paragraph of the proof. Thus $E^{\prime} \supset K$ and, by admissibility, $E^{\prime} / K$ is unramified. The fields $E, E^{\prime}$ are both unramified extensions of $K$ inside $L$, and of the same degree $n /[K: F]$. Therefore $E=E^{\prime}$, and we may assume again that $L / F$ is the Galois closure of $E=E^{\prime}$ over $F$. Applying the analysis of the first paragraph to $\sigma=\operatorname{Ind}_{E / F} \xi$, we get

$$
\tau=\operatorname{Ind}_{E / K} \xi=\operatorname{Ind}_{E / K} \xi^{\prime}
$$


Since $E / K$ is cyclic, this implies that the quasicharacters $\xi, \xi^{\prime}$ of $E^{\times}$are conjugate under $\operatorname{Gal}(E / K)$, whence the pairs $(E / F, \xi),\left(E^{\prime} / F, \xi^{\prime}\right)$ are $F$-isomorphic. Thus (A.3.1) is injective, and we have finished the proof.

A.4. Let $\sigma \in \mathcal{G}_{n}^{0}(F)$; we define $T(\sigma)$ to be the group of unramified characters $\chi$ of $F^{\times}$such that $\chi \otimes \sigma \cong \sigma$. Since $\operatorname{det}(\chi \otimes \sigma)=\chi^{n} \cdot \operatorname{det} \sigma$, the group $T(\sigma)$ is cyclic of order $t(\sigma)$ dividing $n$.

Proposition. Let $(E / F, \xi) \in P_{n}(F)$ and let $\sigma=\operatorname{Ind}_{E / F} \xi$. Then $t(\sigma)=f(E \mid F)$.

Proof. If $\chi$ is an unramified character of $F^{\times}$, let us abbreviate $\chi \circ \mathrm{N}_{E / F}=\chi_{E}$. Then $\chi \otimes \sigma \cong \operatorname{Ind}_{E / F} \xi^{\prime}$, where $\xi^{\prime}=\xi \cdot \chi_{E}$. If $\chi^{f(E \mid F)}=1$, then $\chi_{E}=1$, so we deduce that $f(E \mid F)$ divides $t(\sigma)$.

In the opposite direction, let $\chi \in T(\sigma)$. The pair $\left(E / F, \chi_{E} \xi\right)$ is surely admissible, and so $F$-isomorphic to $(E / F, \xi)$. That is, there exists $\alpha \in \operatorname{Aut}(E \mid F)$ such that $\chi_{E} \xi=\xi^{\alpha}$. Since $\chi_{E}$ is unramified, this implies that $\alpha$ fixes $\xi \mid U_{E}$. If $K$ is the fixed field of $\alpha$, this implies that $\xi \mid U_{E}^{1}$ factors through $\mathrm{N}_{E / K}$, whence $E / K$ is unramified. Now we can deduce that $\xi \mid U_{E}$ factors through $\mathrm{N}_{E / K}$ as well. Any quasicharacter of $E^{\times}$agreeing with $\xi$ on $U_{E}$ therefore factors through $\mathrm{N}_{E / K}$. In particular, this applies to $\xi$. By admissibility, $\alpha=1$, so $\chi_{E}=1$, and $\chi^{f(E \mid F)}=1$, as required.

Corollary. Let $\sigma \in \mathcal{G}_{n}^{0}(F)$. The following conditions are equivalent:

(1) $\sigma$ is essentially tame;

(2) $p$ does not divide $n / t(\sigma)$.

Proof. If $\sigma$ is essentially tame, it is of the form $\operatorname{Ind}_{E / F} \xi$, for some $(E / F, \xi) \in P_{n}(F)$, and so the implication (1) $\Rightarrow(2)$ follows directly from the proposition. Conversely, suppose $p \nmid n / t(\sigma)$. If $E / F$ is unramified of degree $t(\sigma)$, there is an irreducible representation $\tau$ of $\mathcal{W}_{E}$ such that $\sigma \cong \operatorname{Ind}_{E / F} \tau$. The dimension of $\tau$ is prime to $p$ so, when we restrict $\tau$ to the pro- $p$ group $\mathcal{P}_{F}$, it becomes a sum of characters. The same therefore applies to $\sigma$, which is therefore essentially tame.

\section{REFERENCES}

1. J. Arthur and L. Clozel, Simple algebras, base change, and the advanced theory of the trace formula, Annals of Math. Studies, vol. 120, Princeton University Press, 1989. MR1007299 (90m:22041)

2. C.J. Bushnell and A. Fröhlich, Gauss sums and p-adic division algebras, Lecture Notes in Math., vol. 987, Springer, Berlin, Heidelberg, New York, 1983. MR0701540 (84m:12017)

3. C.J. Bushnell and G. Henniart, Local tame lifting for $G L(N)$ I: simple characters, Publ. Math. IHES 83 (1996), 105-233. MR1423022 (98m:11129)

4. MR 1685898 (2000d:11147)

5. , Davenport-Hasse relations and an explicit Langlands correspondence, J. reine angew. Math. 519 (2000), 171-199. Mr.1739725 (2002e:11171)

6. L Local tame lifting for GL(n) IV: simple characters and base change, Proc. London Math. Soc. (3) 87 (2003), 337-362. MR1990931 (2004f:22017)

7. tions, Compositio Mathematica, to appear.

8. C.J. Bushnell, G. Henniart and P.C. Kutzko, Correspondance de Langlands locale pour $\mathrm{GL}_{n}$ et conducteurs de paires, Ann. Scient. École Norm. Sup. (4) 31 (1998), 537-560. MR 1634095 (99h:22011)

9. C.J. Bushnell and P.C. Kutzko, The admissible dual of $G L(N)$ via compact open subgroups, Annals of Math. Studies, vol. 129, Princeton University Press, 1993. MR.1204652 (94h:22007) 
10. The admissible dual of $S L(N)$ II, Proc. London Math. Soc. (3) 68 (1994), 317-379. MR 1253507 (94k:22035)

11. L.J. Corwin and R.E. Howe, Computing characters of tamely ramified p-adic division algebras, Pacific J. Math. 73 (1977), 461-477. MR0492084 (58:11238)

12. G. Glauberman, Correspondences of characters for relatively prime operator groups, Canad. J. Math. 20 (1968), 1465-1488. MR0232866 (38:1189)

13. J.A. Green, The characters of the finite general linear groups, Trans. Amer. Math. Soc. 80 (1955), 402-447. MR0072878(17:345e)

14. M. Harris and R. Taylor, On the geometry and cohomology of some simple Shimura varieties, Annals of Math. Studies, vol. 151, Princeton University Press, 2001. MR 1876802 (2002m:11050)

15. G. Henniart, Une preuve simple des conjectures de Langlands pour $\mathrm{GL}(n)$ sur un corps padique, Invent. Math. 139 (2000), 439-455. MR1738446 (2001e:11052)

16. G. Henniart and R. Herb, Automorphic induction for $G L(n)$ (over local non-archimedean fields), Duke Math. J. 78 (1995), 131-192. MR1328755|(96i:22038)

17. G. Henniart and B. Lemaire, Work in progress.

18. R.E. Howe, Tamely ramified supercuspidal representations of $G L_{n}$, Pacific J. Math. 73 (1977), 437-460. MF 0492087(58:11241)

19. H. Jacquet, I. Piatetski-Shapiro and J. Shalika, Rankin-Selberg convolutions, Amer. J. Math. 105 (1983), 367-483. MR0701565 (85g:11044)

20. H. Koch and E.-W. Zink, Zur Korrespondenz von Darstellungen der Galoisgruppen und der zentralen Divisionsalgebren über lokalen Körpern (der zahme Fall), Math. Nachr. 98 (1980), 83-119. MR0623696 (83h:12025)

21. G. Laumon, M. Rapoport and U. Stuhler, D-elliptic sheaves and the Langlands correspondence, Invent. Math. 113 (1993), 217-338. MR.1228127(96e:11077)

22. C. Mœglin, Sur la correspondance de Langlands-Kazhdan, J. Math. Pures et Appl. (9) 69 (1990), 175-226. MR1067450 (91g:11141)

23. A. Moy, Local constants and the tame Langlands correspondence, Amer. J. Math. 108 (1986), 863-930. MR 0853218 (88b:11081)

24. H. Reimann, Representations of tamely ramified p-adic division and matrix algebras, J. Number Theory 38 (1991), 58-105. MR1105671 (92h:11103)

25. J. Rogawski, Representations of $\mathrm{GL}(n)$ and division algebras over a local field, Duke Math. J. 50 (1983), 161-196. MR0700135 (84j:12018)

26. J.-P. Serre, Corps locaux, Hermann, Paris, 1965. MF 0150130 (27:133)

27. F. Shahidi, Fourier transforms of intertwining operators and Plancherel measures for GL $(n)$, Amer. J. Math. 106 (1984), 67-111. MR0729755 (86b:22031)

Department of Mathematics, King's College London, Strand, London WC2R 2LS, UNITED KINGDOM

E-mail address: bushnell@mth.kcl.ac.uk

Département de Mathématiques \& UMR 8628 du CNRS, BÂtiment 425, Université de Paris-Sud, 91405 Orsay Cedex, France

E-mail address: Guy.Henniart@math.u-psud.fr 